\title{
Real-Time Frequency Response Estimation from Flight Data
}

\author{
Matthew S. Holzel ${ }^{1}$ \\ University of Michigan, Ann Arbor, MI, 48105 \\ Eugene A. Morelli ${ }^{2}$ \\ NASA Langley Research Center, Hampton, VA, 23681
}

\begin{abstract}
A nonparametric method for estimating frequency responses in real-time using a method based on recursive least squares in the time domain was developed and studied. The proposed method uses sinusoidal functions at selected frequencies known to be contained in the input in conjunction with recursive least squares to rapidly estimate Fourier series coefficients for input and output time series, and thereby estimate frequency responses. Practical problems that arise when applying the discrete Fourier transform in recursive form were identified using simple examples. A general expression for accurate real-time calculation of the covariance matrix for recursive least squares parameter estimation was developed and used to calculate valid uncertainty bounds for real-time frequency response estimates. Simulation data generated with optimized multi-sine inputs were used to investigate the accuracy of the proposed method for real-time frequency response estimation, and to validate the calculated error bounds. The approach was also applied in real-time to flight data from a subscale jet transport aircraft. Comparisons of real-time frequency response estimates and error bounds with results from conventional post-flight batch analysis showed that results from the real-time method were in statistical agreement with post-flight batch estimates, with valid error bounds that properly indicated the quality of the real-time frequency response estimates.
\end{abstract}

\begin{tabular}{lll} 
& & \multicolumn{1}{c}{ Nomenclature } \\
ARMAX & $=$ & Autoregressive moving average with exogenous inputs model \\
$F(\cdots)$ & $=$ & Fourier or partial-period Fourier transform \\
$E[\cdots]$ & $=$ & expectation operator \\
$\sigma$ & $=$ & standard deviation on a random variable \\
$j$ & $=$ & imaginary number $=\sqrt{-1}$ \\
$\delta_{e}, \delta_{a}, \delta_{r}$ & $=$ & elevator, aileron, and rudder deflections, rad or deg \\
$\alpha$ & $=$ & angle of attack, rad or deg \\
$q$ & $=$ & body-axis pitch rate, rad/sec or deg/sec \\
$a_{z}$ & $=$ & $z$ body-axis translational accelerometer measurements, $\mathrm{g}$
\end{tabular}

\section{Introduction}

Efficient flight envelope expansion for modern aircraft can be achieved when control system margins can be monitored in real-time, while the aircraft is flying. Control system margins have been monitored in a quasi-realtime fashion using frequency-domain methods on various flight test programs, such as the X-29, X-31, Solar Pathfinder, $\mathrm{X}-36, \mathrm{X}-38$, and $\mathrm{X}-48 \mathrm{~B}^{1-7}$. Taking the approach used in these programs a step further to real-time operation would require real-time estimation of frequency responses, as well as their uncertainty, in the presence of colored output measurement noise. This step toward true real-time estimation of frequency responses is of critical importance when testing control system designs on subscale aircraft, which have compressed flight times and maneuver lengths. Furthermore, real-time estimates of frequency responses and associated uncertainties would be very useful for monitoring the dynamics and control of unusual vehicles in flight, or to characterize the dynamics and control of a damaged aircraft for reconfigurable control. In such cases, a parametric model structure might be in

\footnotetext{
${ }^{1} \mathrm{PhD}$ candidate in Aerospace Engineering, NASA ASP Fellow

${ }^{2}$ Research Engineer, Dynamic Systems and Control Branch, MS 308, Associate Fellow
} 
doubt or difficult to ascertain. Some work has been done on this problem in the past using the Ho-Kalman approach to modeling in discrete-time ${ }^{8,9}$, as well as recursive discrete-time ARMAX $^{10}$. This paper investigates the efficacy of a new approach based on applying recursive least squares in the time domain to estimate Fourier coefficients. Accurate uncertainty estimates are estimated, then used to compute uncertainty in real-time estimates of frequency responses. Throughout the paper, it is assumed that the input to the system is a known multi-sine and that there exists zero-mean, possibly colored output measurement noise.

In the first section, practical issues associated with implementing the Fourier transform in real-time are examined, namely, since the Fourier integral can be updated recursively, the result of evaluating the Fourier integral at non-integer multiples of the signal period is analyzed. In the second section, it is shown how least-squares can be used to estimate the Fourier transform, and a general framework for batch least-squares and a method for estimating the covariance of the least-squares estimates in the presence of zero-mean, possibly colored output measurement noise are presented. The next section shows how both the least-squares estimates and their covariance can be updated recursively. Following that, it is shown how to convert uncertainty in the least-squares estimates to uncertainty in the magnitude and phase of the frequency response estimate. Simulation results are then presented for a longitudinal aircraft model, where the recursive least-squares estimates and estimated covariance for the frequency response are compared to the true system. In the simulation, a realization of colored noise representative of model residuals due to unmodeled nonlinearities is used to corrupt the output signal. Finally, the method is applied to flight data from the T-2 subscale jet transport aircraft, where the results are compared with traditional batch post-flight analysis, followed by concluding remarks that summarize the results and their significance.

\section{Practical Aspects of the Fourier Transform}

Use of the Fourier transform is common in the system identification literature and remains the basis for most nonparametric system identification techniques. However, practical issues arise when trying to implement the Fourier transform in real-time. Specifically, because the Fourier integral can be updated recursively, the result of evaluating the Fourier integral at non-integer multiples of the signal period needs to be analyzed. This is also important for quick estimation because estimates of the Fourier transform are desired before a complete period of the signal has concluded. The analysis begins by introducing the Fourier transform and the partial-period Fourier transform.

\section{A. Fourier Transform}

Consider the signal $f(t)$, which is a sum of harmonic sinusoids given by

$$
f(t)=\sum_{i=1}^{N} a_{i} \cos \left(\frac{2 \pi i}{T} t+\phi_{i}\right)
$$

where $T$ is the time length, $N / T$ is the highest frequency component, and $a_{i}$ may be zero for some $i \in[1, N]$. Then the Fourier transform $F(v)$ of $f(t)$ is given by

$$
F(v) \triangleq \frac{2}{T} \int_{0}^{T} f(t)[\cos (2 \pi v t)-j \sin (2 \pi v t)] d t=\frac{2}{T} \int_{0}^{T} f(t) e^{-j(2 \pi v t)} d t
$$

Furthermore, the partial-period Fourier transform $F(v, a, b)$ of $f(t)$ is given by

$$
F(v, a, b) \triangleq \frac{2}{b-a} \int_{a}^{b} f(t)[\cos (2 \pi v t)-j \sin (2 \pi v t)] d t=\frac{2}{b-a} \int_{a}^{b} f(t) e^{-j(2 \pi v t)} d t
$$

Letting $v$ be an integer multiple of $1 / T$, that is, $v=i / T$, it follows that the Fourier transform evaluated at the frequency $v$ is given by

$$
F(v)=\left\{\begin{aligned}
a_{i} e^{j \phi_{i}}, & 1 \leq i \leq N \\
0, & i>N
\end{aligned}\right.
$$

where the magnitude of $F(v)$ is $a_{i}$ and the argument of $F(v)$ is $\phi_{i}$.

Note that the definition of the Fourier transform requires that the fundamental frequency, or equivalently, the period, of $f(t)$ is known. When $v$ is not an integer multiple of the fundamental frequency $1 / T$, Eq. (2) does not hold. In fact, $F(v)$ will in general be nonzero, even when $f(t)$ has no frequency content at the frequency $v$. 


\section{B. Partial-Period Fourier Transform}

Recursively updating the Fourier integral in real-time is tantamount to computing the partial-period Fourier transform. It is therefore important to know if the partial-period Fourier transform is a reliable estimate of the Fourier transform. Unfortunately, the following example demonstrates that the partial-period Fourier transform is not a reliable estimate of the Fourier transform, and that the partial-period Fourier transform will in general be nonzero at off-nominal frequencies and have errors even at the nominal frequencies. However, the errors become smaller as the time length of the data increases.

Example 1 Let $f(t)=a \cos \left(2 \pi f_{0} t+\phi\right)$, and consider the partial-period Fourier transform of $f(t)$ evaluated at $f_{0} \mathrm{~Hz}$ and $k f_{0} \mathrm{~Hz}$, where $k \neq 1$, that is,

$$
\begin{gathered}
F\left(f_{0}, 0, t\right)=\frac{2}{t} \int_{0}^{t} a \cos \left(2 \pi f_{0} \tau+\phi\right)\left[\cos \left(2 \pi f_{0} \tau\right)-j \sin \left(2 \pi f_{0} \tau\right)\right] d \tau \\
F\left(k f_{0}, 0, t\right)=\frac{2}{t} \int_{0}^{t} a \cos \left(2 \pi f_{0} \tau+\phi\right)\left[\cos \left(2 \pi k f_{0} \tau\right)-j \sin \left(2 \pi k f_{0} \tau\right)\right] d \tau
\end{gathered}
$$

Then the real and imaginary parts of $F\left(f_{0}, 0, t\right)$ and $F\left(k f_{0}, 0, t\right)$ are given by

$$
\begin{gathered}
\operatorname{Re}\left[F\left(f_{0}, 0, t\right)\right]=\frac{a}{4 \pi f_{0} t}\left[4 \pi f_{0} t \cos \phi+\sin \left(4 \pi f_{0} t+\phi\right)-\sin \phi\right] \\
\operatorname{Im}\left[F\left(f_{0}, 0, t\right)\right]=\frac{a}{4 \pi f_{0} t}\left[4 \pi f_{0} t \sin \phi+\cos \left(4 \pi f_{0} t+\phi\right)-\cos \phi\right] \\
\operatorname{Re}\left[F\left(k f_{0}, 0, t\right)\right]=\frac{a}{2 \pi f_{0} t\left(k^{2}-1\right)}\left[(k-1) \sin \left(2 \pi[k+1] f_{0} t+\phi\right)+(k+1) \sin \left(2 \pi[k-1] f_{0} t-\phi\right)+2 \sin \phi\right] \\
\operatorname{Im}\left[F\left(k f_{0}, 0, t\right)\right]=\frac{a}{2 \pi f_{0} t\left(k^{2}-1\right)}\left[(k-1) \cos \left(2 \pi[k+1] f_{0} t+\phi\right)+(k+1) \cos \left(2 \pi[k-1] f_{0} t-\phi\right)-2 k \cos \phi\right]
\end{gathered}
$$

From the preceding equations, the partial-period Fourier transform is seen to be generally inaccurate; that is, at the nominal frequency $f_{0}$, typically $F\left(f_{0}, 0, t\right) \neq F\left(f_{0}\right)=a e^{j \phi}$, and at off-nominal frequencies, typically $F\left(k f_{0}, 0, t\right) \neq F\left(k f_{0}\right)=0$. Specifically, for all $k>1$ and $t>0$, the partial-period Fourier transform errors are bounded by

$$
\begin{aligned}
& \left|F\left(f_{0}, 0, t\right)-a e^{j \phi}\right| \leq \frac{a}{2 \pi f_{0} t} \\
& \left|F\left(k f_{0}, 0, t\right)\right| \leq \frac{2 a k}{\pi f_{0} t\left(k^{2}-1\right)}
\end{aligned}
$$

where the error bounds, and hence the true errors, approach 0 as both $t$ and $k$ increase, that is,

$$
\begin{gathered}
\lim _{t \rightarrow \infty}\left[F\left(f_{0}, 0, t\right)-a e^{j \phi}\right]=0 \\
\lim _{t \rightarrow \infty} F\left(k f_{0}, 0, t\right)=0 \\
\lim _{k \rightarrow \infty} F\left(k f_{0}, 0, t\right)=0
\end{gathered}
$$

The upper bound for the error in the partial-period Fourier transform is proportional to $1 / t$, which in some cases may be unacceptably slow convergence. Figure 1 displays the bounds for $a=1, f_{0}=1 \mathrm{~Hz}$, and various $k$ and $t$. Figure 2 compares the magnitude of the true partial-period errors to the bounds given by Eqs. (3) and (4) when $a=1, f_{0}=1 \mathrm{~Hz}, \phi=0 \mathrm{rad}$, and $k>1$. Several $k$ are chosen to exemplify the errors and bounds when evaluating the partial-period Fourier transform at the nominal frequency $(k=1)$, a harmonic multiple of the nominal frequency $(k=5)$, a non-harmonic multiple of the nominal frequency $(k=\pi)$, and at a very high frequency compared to the nominal $(k=100)$. Note that when $f(t)$ is composed of more than one sinusoid, the partial-period errors are additive. In Figure 2, the shading that appears for $k=100$ is due to high frequency oscillation of the error. 


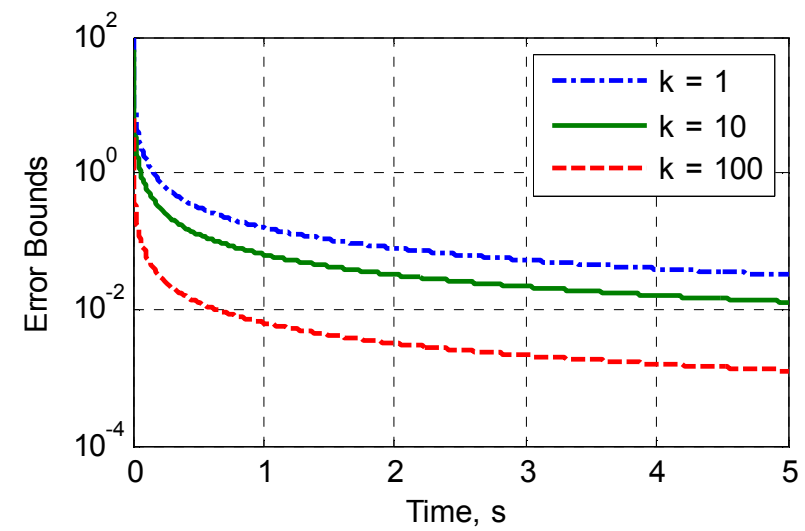

Figure 1. Partial-period Fourier transform error bounds for $a=1, f_{0}=1 \mathrm{~Hz}$, and various $k$ and $t$
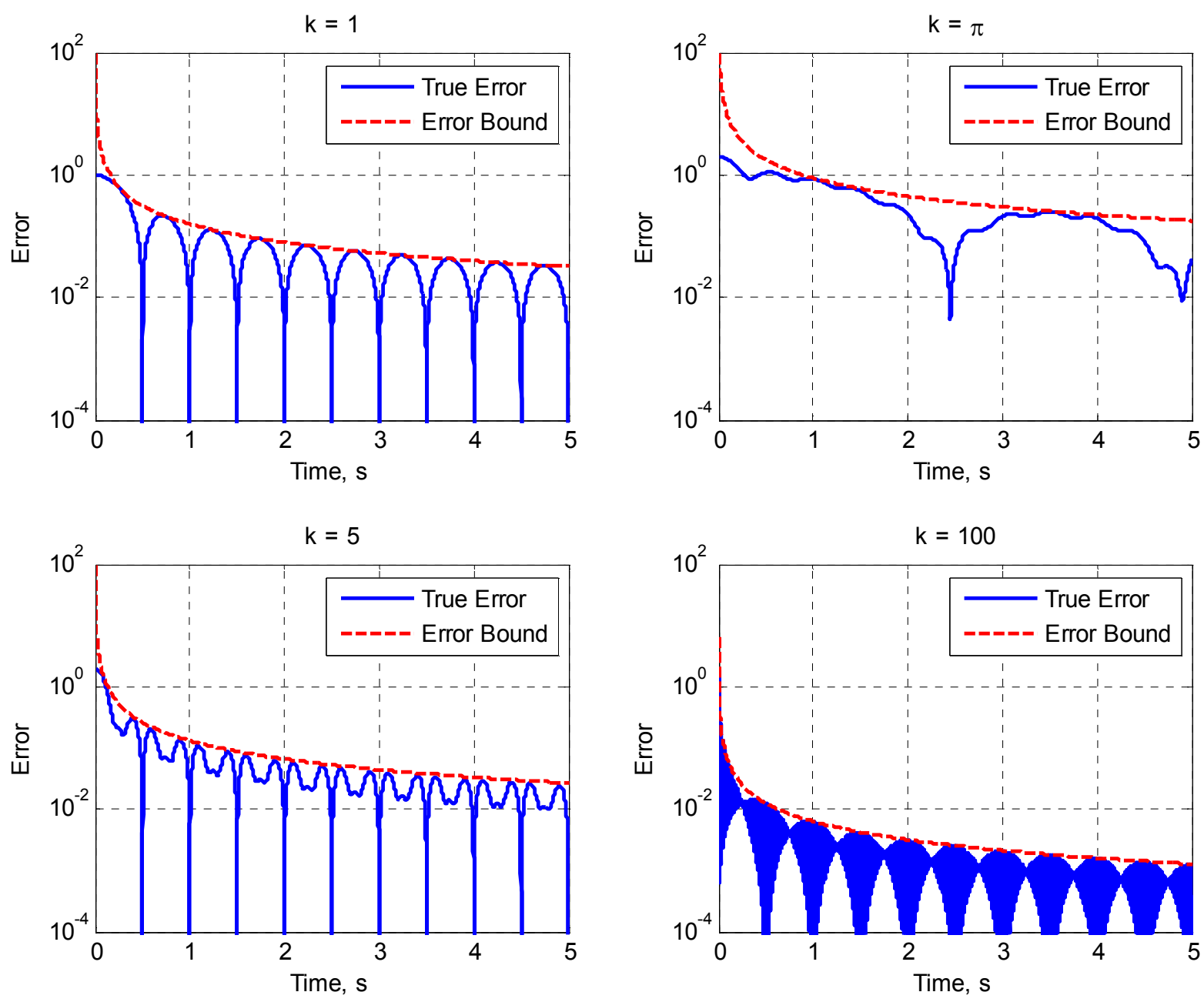

Figure 2. Comparison of the true partial-period Fourier transform errors to the bounds given by Eqs. (3)-(4) for $a=1, f_{0}=1 \mathrm{~Hz}, \phi=0 \mathrm{rad}$, and various $k>1$ 


\section{Least-Squares Estimates of the Fourier Transform}

In the previous section, some of the deficiencies of the partial-period Fourier transform were demonstrated. However, it is desirable to have estimates of the Fourier transform in real-time, without having to wait for integer periods. Hence least-squares is used here to estimate the magnitude and phase of the output indirectly at specified frequencies and non-integer periods of the signal. Furthermore, from the approximate linearity of the system and the assumption that the input is a known multi-sine, the frequencies at which the output will have content are known a priori.

\section{A. Estimation of the Fourier Transform}

Estimation of the unknown coefficients of a model, such as the amplitudes $a_{1}, \ldots, a_{N}$ and phases $\phi_{1}, \ldots, \phi_{N}$ in Eq. (1) is, in general, difficult since the model is nonlinear in the model coefficients. Here, the unknown amplitudes and phases are estimated indirectly, by first recasting the model of Eq. (1) into a model which is linear in the parameters. Specifically, let the output $y(t)$ be given by Eq. (1) and let $\beta_{i} \triangleq a_{i} \cos \phi_{i}$ and $\gamma_{i} \triangleq a_{i} \sin \phi_{i}$ for all $i=1, \ldots, N$. Then

$$
y(t)=\sum_{i=1}^{N} \beta_{i} \cos \left(\frac{2 \pi i}{T} t\right)-\gamma_{i} \sin \left(\frac{2 \pi i}{T} t\right)=\theta x(t)
$$

where $\theta$ and $x(t)$ are given by

$$
\begin{gathered}
\theta \triangleq\left[\begin{array}{llllll}
\beta_{1} & \ldots & \beta_{N} & \gamma_{1} & \ldots & \gamma_{N}
\end{array}\right] \\
x(t) \triangleq\left[\begin{array}{llllll}
\cos \left(\frac{2 \pi}{T} t\right) & \ldots & \cos \left(\frac{2 \pi N}{T} t\right) & -\sin \left(\frac{2 \pi}{T} t\right) & \ldots & -\sin \left(\frac{2 \pi N}{T} t\right)
\end{array}\right]^{T}
\end{gathered}
$$

Next, let $z(t)$ denote a measurement of $y(t)$ which is corrupted by additive zero-mean colored measurement noise,

$$
z(t)=y(t)+v(t)=\theta x(t)+v(t)
$$

Then $\theta$ can be estimated using least-squares when $z(t)$ is sampled. Furthermore, once estimates of $\beta_{i}$ and $\gamma_{i}$ are obtained, estimates of $a_{i}$ and $\phi_{i}$ can be found from

$$
\begin{aligned}
a_{i} & =\sqrt{\beta_{i}^{2}+\gamma_{i}^{2}} \\
\phi_{i} & =\tan ^{-1}\left(\frac{\gamma_{i}}{\beta_{i}}\right)
\end{aligned}
$$

\section{B. Least-Squares Estimates and Their Covariance}

Ordinary least-squares and its recursive version are well-understood algorithms. Furthermore, when possibly colored output measurement noise is present, the least-squares estimates themselves are random, and it is possible to estimate the covariance of the least-squares estimates offline ${ }^{11,12}$. In this section, it is shown how to compute an estimate for the covariance of ordinary least-squares estimates in batch mode, in the presence of zero-mean, possibly colored output measurement noise. This material is based on work documented in Refs. [11] and [12]. The next section shows how this approach can be implemented recursively for real-time operations.

Begin by considering the linear model

$$
y(t)=a_{1} x_{1}(t)+\cdots+a_{r} x_{r}(t)
$$

or simply $y(t)=\theta x(t)$, where $\theta \triangleq\left[\begin{array}{lll}a_{1} & \ldots & a_{r}\end{array}\right]$ and $x(t)=\left[\begin{array}{lll}x_{1}(t) & \ldots & x_{r}(t)\end{array}\right]^{T}$. Furthermore, for simplicity of the argument, let $x(t)$ be deterministic.

Next, let $z(t)$ denote a measurement of $y(t)$ which is corrupted by additive zero-mean colored measurement noise,

$$
z(t)=y(t)+v(t)=\theta x(t)+v(t)
$$

where $v$ is a realization of the random, stationary, zero-mean, colored noise process $\mathcal{V}$. Then, letting $z(t)$ be known at the uniformly spaced times $t_{1}=t_{0}+\Delta, \ldots, t_{n}=t_{0}+n \Delta$, it follows that 


$$
Z_{n}=\theta \mathrm{X}_{n}+V_{n}
$$

where $Z_{n} \triangleq\left[\begin{array}{lll}z\left(t_{1}\right) & \ldots & z\left(t_{n}\right)\end{array}\right], \mathrm{X}_{n} \triangleq\left[\begin{array}{lll}x\left(t_{1}\right) & \ldots & x\left(t_{n}\right)\end{array}\right]$, and $V_{n} \triangleq\left[\begin{array}{llll}v\left(t_{1}\right) & \ldots & v\left(t_{n}\right)\end{array}\right]$. Furthermore, if the product $X_{n} X_{n}^{T}$ is nonsingular, then $\theta$, the least-squares estimate $\hat{\theta}_{n}$ of $\theta$, and the covariance of $\hat{\theta}_{n}$, denoted by $P\left(\hat{\theta}_{n}\right)$, are given by

$$
\begin{gathered}
\theta=Z_{n} \mathrm{X}_{n}^{T} \Gamma_{n}-V_{n} \mathrm{X}_{n}^{T} \Gamma_{n} \\
\hat{\theta}_{n}=Z_{n} \mathrm{X}_{n}^{T} \Gamma_{n} \\
P\left(\hat{\theta}_{n}\right)=\mathrm{E}\left[\Gamma_{n} \mathrm{X}_{n} V_{n}^{T} V_{n} \mathrm{X}_{n}^{T} \Gamma_{n}\right]
\end{gathered}
$$

where $\Gamma_{n} \triangleq\left(\mathrm{X}_{n} \mathrm{X}_{n}^{T}\right)^{-1}$ and, since $\mathrm{X}_{n}$ is deterministic,

$$
P\left(\hat{\theta}_{n}\right)=\Gamma_{n} \mathrm{X}_{n} \mathrm{E}\left[V_{n}^{T} V_{n}\right] \mathrm{X}_{n}^{T} \Gamma_{n}
$$

Note that the least-squares estimate $\hat{\theta}_{n}$ is unbiased since

$$
\begin{aligned}
\mathrm{E}\left[\hat{\theta}_{n}\right] & =\mathrm{E}\left[Z_{n} \mathrm{X}_{n}^{T} \Gamma_{n}\right] \\
& =\mathrm{E}\left[\left(\theta \mathrm{X}_{n}+V_{n}\right) \mathrm{X}_{n}^{T} \Gamma_{n}\right] \\
& =\theta \mathrm{X}_{n} \mathrm{X}_{n}^{T} \Gamma_{n}+\mathrm{E}\left[V_{n}\right] \mathrm{X}_{n}^{T} \Gamma_{n} \\
& =\theta+\mathrm{E}\left[V_{n}\right] \mathrm{X}_{n}^{T} \Gamma_{n} \\
& =\theta
\end{aligned}
$$

Finally, note that $\mathrm{E}\left[V_{n}^{T} V_{n}\right]$ is the autocorrelation matrix of $\mathcal{V}$,

$$
\mathrm{E}\left[V_{n}^{T} V_{n}\right] \triangleq R_{v v}^{n}=\left[\begin{array}{ccc}
R_{v v}(0) & \cdots & R_{v v}([n-1] \Delta) \\
\vdots & \ddots & \vdots \\
R_{v v}([n-1] \Delta) & \cdots & R_{v v}(0)
\end{array}\right]
$$

where $R_{v v}(i \Delta) \triangleq \mathrm{E}\left[v^{T}(t) v(t+i \Delta)\right]$. Furthermore, letting $\hat{v}(t) \triangleq z(t)-\hat{\theta}_{n} x(t)$ for all $t=t_{1}, \ldots, t_{n}$ yields the estimated autocorrelation matrix

where

$$
\hat{R}_{v v}^{n} \triangleq\left[\begin{array}{ccc}
\hat{R}_{v v}^{n}(0) & \cdots & \hat{R}_{v v}^{n}([n-1] \Delta) \\
\vdots & \ddots & \vdots \\
\hat{R}_{v v}^{n}([n-1] \Delta) & \cdots & \hat{R}_{v v}^{n}(0)
\end{array}\right]
$$

$$
\hat{R}_{v v}^{n}(i \Delta)=\left(\frac{1}{n}\right) \sum_{j=1}^{n-i} \hat{v}^{T}\left(t_{j}\right) \hat{v}\left(t_{j}+i \Delta\right)
$$

Hence the estimated covariance of $\hat{\theta}_{n}$, denoted by $\widehat{P}\left(\hat{\theta}_{n}\right)$, is given by

$$
\widehat{P}\left(\hat{\theta}_{n}\right)=\Gamma_{n} \mathrm{X}_{n} \hat{R}_{v v}^{n} \mathrm{X}_{n}^{T} \Gamma_{n}
$$

Note that the usual definition of $\hat{R}_{v v}^{n}(i \Delta)$ is $\left(\frac{1}{n-i}\right) \sum_{j=1}^{n-i} \hat{v}^{T}\left(t_{j}\right) \hat{v}\left(t_{j}+i \Delta\right)$. However, for large $i, \hat{R}_{v v}^{n}(i \Delta)$ is dependent on only a few samples, and is therefore not a good estimate. The definition given above resolves this effect by noting that the autocorrelation function usually tends to 0 for large $i$ anyway. Also, for large $n$ and moderate $i, n \approx n-i$, that is, for moderate lags, the above definition approaches the usual one as the amount of data increases.

Example 2 Consider the colored noise sequence

$$
v(k)=0.2 w(k)+0.1 w(k-1)-0.02 w(k-2)-0.01 w(k-3)
$$

where $w$ is a realization of the zero-mean stationary white Gaussian process $\mathcal{W}$ with variance $\sigma_{w}^{2}=10$. Then the autocorrelation function of $\mathcal{V}$ is given by

$$
R_{v v}(i \Delta)=\left\{\begin{aligned}
+0.0505 \sigma_{w}^{2}, & |i|=0 \\
+0.0182 \sigma_{w}^{2}, & |i|=1 \\
-0.0050 \sigma_{w,}^{2}, & |i|=2 \\
-0.0020 \sigma_{w,}^{2}, & |i|=3 \\
0, & |i| \geq 4
\end{aligned}\right.
$$


Also, consider the discrete-time system

$$
z(k)=u(k)-0.7 u(k-1)+0.3 u(k-2)-0.1 u(k-3)+v(k)
$$

where $z(k)$ is the measured output, $u(k)$ is the known input, and $v(k)$ is an unmeasured colored noise sequence. Then $z(k)=\theta x(k)+v(k)$, where $\theta$ and $x(k)$ are given by

$$
\begin{aligned}
\theta & =\left[\begin{array}{llll}
+1.0 & -0.7 & +0.3 & -0.1
\end{array}\right] \\
x(k) & =\left[\begin{array}{llll}
u(k) & u(k-1) & u(k-2) & u(k-3)
\end{array}\right]^{T}
\end{aligned}
$$

Furthermore, let $u$ be a Schroeder-phased signal ${ }^{13}$ given by

$$
u(k)=\sum_{i=1}^{N} \cos \left(\left[\frac{2 \pi i}{T}\right] k-\frac{\pi i^{2}}{N}\right)
$$

where $N=20$ and $T=200$. Then as described previously, for each fixed value of $n$ and each realization of the colored noise process $\mathcal{V}$, one can compute the least-squares estimate $\hat{\theta}_{n}=Z_{n} X_{n}^{T}\left(X_{n} X_{n}^{T}\right)^{-1}$ and the estimated autocorrelation matrix $\hat{R}_{v v}^{n}$. Considering 10,000 realizations of the colored noise process $\mathcal{V}$, and computing the estimated correlation matrix for each $j=1, \ldots, 10,000$, denoted by $\hat{R}_{v v, j}^{n}$, yields the sample mean squared error in the estimated autocorrelation matrix

$$
\epsilon_{n}(i \Delta)=\frac{1}{10,000} \sum_{j=1}^{10,000}\left(\hat{R}_{v v, j}^{n}(i \Delta)-R_{v v}(i \Delta)\right)^{2}
$$

Figure 3 displays the sample mean squared error $\epsilon_{n}(i \Delta)$ for $i=0, \ldots, 5$ and various $n$, from which it appears that as $n$ increases, the estimates $\hat{R}_{v v}^{n}(i \Delta)$ approach $R_{v v}(i \Delta)$, that is, $\hat{R}_{v v}^{n}(i \Delta)$ appears to be a consistent estimate of $R_{v v}(i \Delta)$. Furthermore, since the covariance estimate $\hat{P}\left(\hat{\theta}_{n}\right)$ is linear in $\hat{R}_{v v}^{n}$, it follows that $\hat{P}\left(\hat{\theta}_{n}\right)$ is a consistent estimate of $P\left(\hat{\theta}_{n}\right)$.

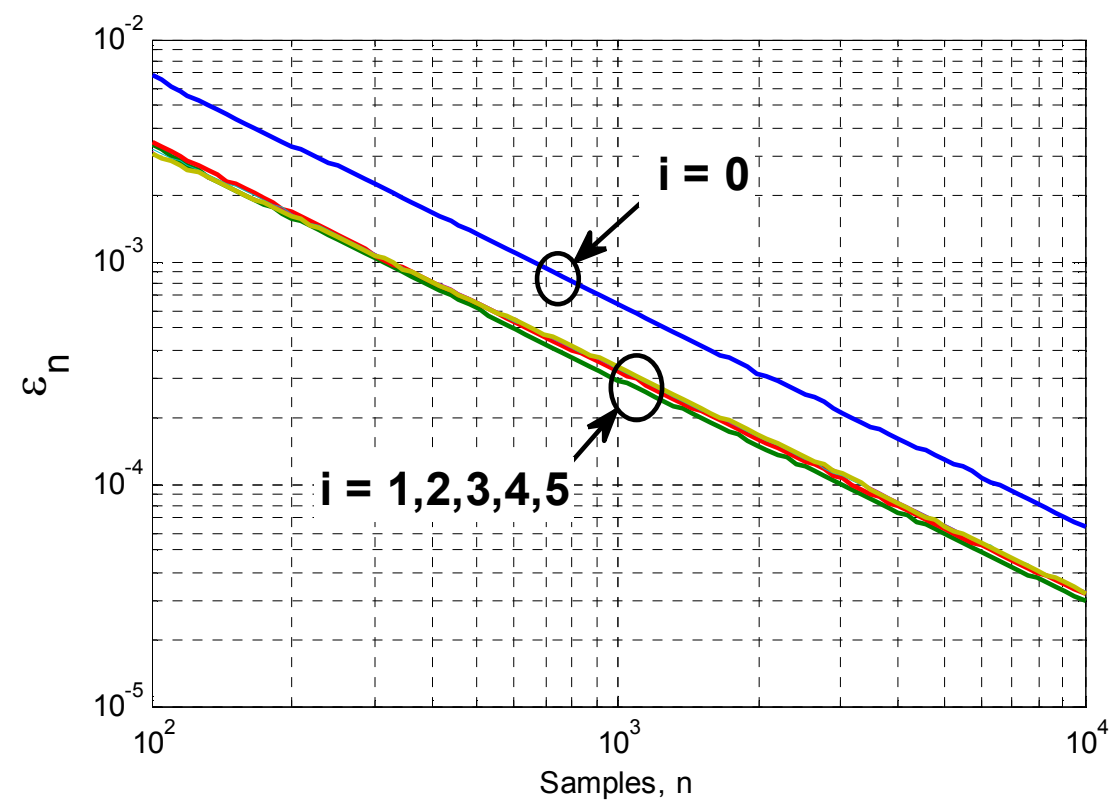

Figure 3. Sample mean squared error in estimated autocorrelation matrix for $i=0, \ldots, 5$ and various $n$

One major impediment to using recursive least-squares in the time domain is that there is no method for accurately computing the covariance of the estimates $\hat{\theta}_{n}$ in real-time for colored noise. The next section shows how this can be achieved. 


\section{Recursive Least-Squares Estimates and Covariance}

In the previous section, a framework for least-squares was developed and a method for estimating the covariance of least-squares estimates was given for colored noise. Here it is shown how both the least-squares estimates and the estimate of their covariance can be updated recursively by approximating the autocorrelation of the noise with a finite number of terms.

First, suppose that $\hat{\theta}_{n}, \Gamma_{n}, z\left(t_{n+1}\right)$, and $x\left(t_{n+1}\right)$ are known, that is, all of the data up to time $t_{n+1}$ are known, including the previous estimate $\hat{\theta}_{n}$ and the inverse of $X_{n} X_{n}^{T}$, denoted by $\Gamma_{n}$. Then the updated least-squares estimate $\hat{\theta}_{n+1}$ is given by ${ }^{11}$

$$
\hat{\theta}_{n+1}=\hat{\theta}_{n}\left[I-x\left(t_{n+1}\right) k\left(t_{n+1}\right)\right]+z\left(t_{n+1}\right) k\left(t_{n+1}\right)
$$

where $I$ is the identity matrix and $k\left(t_{n+1}\right)$ is given by

$$
k\left(t_{n+1}\right)=\frac{x^{T}\left(t_{n+1}\right) \Gamma_{n}}{1+x^{T}\left(t_{n+1}\right) \Gamma_{n} x\left(t_{n+1}\right)}
$$

Furthermore, from the matrix inversion lemma, the inverse of $X_{n+1}^{T} X_{n+1}$ is given in terms of the previous inverse $\Gamma_{n}=\left(X_{n}^{T} X_{n}\right)^{-1}$ by

$$
\Gamma_{n+1}=\left(\mathrm{X}_{n+1} \mathrm{X}_{n+1}^{T}\right)^{-1}=\Gamma_{n}\left[I-x\left(t_{n+1}\right) k\left(t_{n+1}\right)\right]
$$

Next, note that the estimated covariance of $\hat{\theta}_{n}$, denoted by $\widehat{P}\left(\hat{\theta}_{n}\right)$, is given by ${ }^{11,12}$

$$
\hat{P}\left(\hat{\theta}_{n}\right)=\Gamma_{n}\left[\sum_{i=1}^{n} \sum_{j=1}^{n} x\left(t_{i}\right) \hat{R}_{v v}^{n}([i-j] \Delta) x^{T}\left(t_{j}\right)\right] \Gamma_{n}
$$

Letting the autocorrelation of $\mathcal{V}, R_{v v}$, be approximated by the first $s+1$ terms, it follows that

$$
\hat{P}\left(\hat{\theta}_{n}\right) \approx \Gamma_{n}\left[\sum_{i=0}^{s} \hat{R}_{v v}^{n}(i \Delta) \Lambda_{n}(i)\right] \Gamma_{n}
$$

where $\Lambda_{n}(i)$ is given by

$$
\Lambda_{n}(i)=\left\{\begin{array}{rr}
\sum_{j=1}^{n} x\left(t_{j}\right) x^{T}\left(t_{j}\right), & i=0 \\
\sum_{j=1}^{n-i} x\left(t_{i+j}\right) x^{T}\left(t_{j}\right)+x\left(t_{j}\right) x^{T}\left(t_{i+j}\right), & i>0
\end{array}\right.
$$

and $\Lambda_{n}(i)$ can be updated recursively since

$$
\Lambda_{n+1}(i)=\left\{\begin{aligned}
\Lambda_{n}(0)+x\left(t_{n+1}\right) x^{T}\left(t_{n+1}\right), & i=0 \\
\Lambda_{n}(i)+x\left(t_{n+1}\right) x^{T}\left(t_{n+1-i}\right)+x\left(t_{n+1-i}\right) x^{T}\left(t_{n+1}\right), & i>0
\end{aligned}\right.
$$

Furthermore, since $\hat{v}(t) \triangleq z(t)-\hat{\theta}_{n} x(t)$, for each $i=0, \ldots, s$, it follows that

where

$$
\hat{R}_{v v}^{n}(i \Delta)=\frac{1}{n}\left(A_{n}(i)-B_{n}(i) \operatorname{vec}\left[\hat{\theta}_{n}\right]+C_{n}(i) \operatorname{vec}\left[\hat{\theta}_{n}^{T} \hat{\theta}_{n}\right]\right)
$$

$$
\begin{aligned}
& A_{n}(i) \triangleq \sum_{j=1}^{n-i} z^{T}\left(t_{j}\right) z\left(t_{i+j}\right) \\
& B_{n}(i) \triangleq \sum_{j=1}^{n-i}\left[x^{T}\left(t_{i+j}\right) \otimes z^{T}\left(t_{j}\right)\right]+\left[x^{T}\left(t_{j}\right) \otimes z^{T}\left(t_{i+j}\right)\right]
\end{aligned}
$$




$$
C_{n}(i) \triangleq \sum_{j=1}^{n-i} x^{T}\left(t_{i+j}\right) \otimes x^{T}\left(t_{j}\right)
$$

and $A_{n}(i), B_{n}(i)$, and $C_{n}(i)$ can be updated recursively since

$$
\begin{aligned}
& A_{n+1}(i)=A_{n}(i)+z^{T}\left(t_{n+1-i}\right) z\left(t_{n+1}\right) \\
& B_{n+1}(i)=B_{n}(i)+\left[x^{T}\left(t_{n+1}\right) \otimes z^{T}\left(t_{n+1-i}\right)\right]+\left[x^{T}\left(t_{n+1-i}\right) \otimes z^{T}\left(t_{n+1}\right)\right] \\
& C_{n+1}(i)=C_{n}(i)+\left[x^{T}\left(t_{n+1}\right) \otimes x^{T}\left(t_{n+1-i}\right)\right]
\end{aligned}
$$

Refer to Appendix A for a discussion of the vec operator and Kronecker product $\otimes$.

Combining Eqs. (5)-(13), the updated estimate $\hat{\theta}_{n+1}$ and its estimated covariance $\hat{P}\left(\hat{\theta}_{n}\right)$ can be calculated recursively. The following algorithm summarizes the approach for updating the least-squares estimates and the estimate of their covariance. It is assumed that $\hat{\theta}_{n}, \Gamma_{n}, z\left(t_{n+1}\right), x\left(t_{n+1}\right), \Lambda_{n}(0), \ldots, \Lambda_{n}(s), A_{n}(0), \ldots, A_{n}(s)$, $B_{n}(0), \ldots, B_{n}(s)$, and $C_{n}(0), \ldots, C_{n}(s)$ are known, and that the noise autocorrelation can be approximated by the first $s+1$ terms.

\section{Algorithm for Recursively Estimating $\widehat{\boldsymbol{\theta}}_{n+1}$ and $\widehat{\boldsymbol{P}}\left(\widehat{\boldsymbol{\theta}}_{n+1}\right)$ for Colored Noise}

Calculate $k\left(t_{n+1}\right)$ from Eq. (6).

Compute the updated least-squares estimate $\hat{\theta}_{n+1}$ using Eq. (5).

Calculate $\Gamma_{n+1}$ using Eq. (7).

Compute $\Lambda_{n+1}(0), \ldots, \Lambda_{n+1}(s)$ using Eq. (9).

Calculate $A_{n+1}(0), \ldots, A_{n+1}(s), B_{n+1}(0), \ldots, B_{n+1}(s)$, and $C_{n+1}(0), \ldots, C_{n+1}(s)$ using Eqs. (11)-(13).

Compute $\hat{R}_{v v}^{n+1}$ using Eq. (10).

Calculate the estimated covariance of $\hat{\theta}_{n+1}, \hat{P}\left(\hat{\theta}_{n+1}\right)$, using Eq. (8).

\section{Frequency Response Estimates and Uncertainty Conversion}

In the previous two sections, a method for computing recursive least-squares estimates and an estimate of their covariance in the presence of possibly colored measurement noise was developed. It was also shown how to make the Fourier transform model a linear model, namely, by computing the least-squares estimates $\hat{\beta}_{i}=a_{\imath} \widehat{\cos } \phi_{l}$ and $\hat{\gamma}_{i}=a_{\iota} \widehat{\sin } \phi_{\iota}$ instead of directly computing the magnitude $a_{i}$ and phase $\phi_{i}$ of the frequency components. Here it is shown how estimates of the magnitude and phase of the frequency response are computed, along with their variances, given $\hat{\beta}_{i}, \hat{\gamma}_{i}$, and the covariance matrix of $\hat{\beta}_{i}$ and $\hat{\gamma}_{i}$.

First, let the output $y(t)$ and input $u(t)$ be given by

$$
\begin{aligned}
& y(t)=\sum_{i=1}^{N} a_{i} \cos \left(\frac{2 \pi i}{T} t+\phi_{i}\right) \\
& u(t)=\sum_{i=1}^{N} b_{i} \cos \left(\frac{2 \pi i}{T} t+\eta_{i}\right)
\end{aligned}
$$

Then the frequency response $G(v)$ between the input and output is the ratio of the Fourier transform of the output $Y(v)$ to the Fourier transform of the input $U(v)$. Letting $v=i / T, G(v)$ is given by

$$
G(v)=\frac{Y(v)}{U(v)}=\frac{a_{i} e^{j \phi_{i}}}{b_{i} e^{j \eta_{i}}}=\left(\frac{a_{i}}{b_{i}}\right) e^{j\left(\phi_{i}-\eta_{i}\right)}
$$

When the input is known, the estimated frequency response is given by 


$$
\widehat{G}(v)=\left(\frac{\hat{a}_{i}}{b_{i}}\right) e^{j\left(\widehat{\phi}_{i}-\eta_{i}\right)}
$$

where $\hat{a}_{i}$ and $\hat{\phi}_{i}$ are computed for the output $y(t)$ from the least-squares estimates $\hat{\beta}_{i}$ and $\hat{\gamma}_{i}$, specifically,

$$
\begin{aligned}
& \hat{a}_{i}=\sqrt{\hat{\beta}_{i}^{2}+\hat{\gamma}_{i}^{2}} \\
& \hat{\phi}_{i}=\tan ^{-1}\left(\frac{\hat{\gamma}_{i}}{\hat{\beta}_{i}}\right)
\end{aligned}
$$

Next, recall that $\hat{\theta}_{n}$ is an unbiased estimate of $\theta$. Hence $\hat{\beta}_{i}$ and $\hat{\gamma}_{i}$ are unbiased estimates of $\beta_{i}$ and $\gamma_{i}$, and it follows that the variance $P\left(\hat{a}_{i}\right)$ is given by

$$
P\left(\hat{a}_{i}\right)=P\left(\hat{\beta}_{i}\right)+P\left(\hat{\gamma}_{i}\right)+2\left(a_{i}-\mathrm{E}\left[\hat{a}_{i}\right]\right) a_{i}
$$

where $a_{i}-\mathrm{E}\left[\hat{a}_{i}\right]$ is the bias in the estimate of $a_{i}$. Assuming that $\hat{a}_{i}$ is an unbiased estimate of $a_{i}$, the variance in the magnitude of the estimated frequency response, $\hat{a}_{i} / b_{i}$, is thus given by

$$
P\left(\frac{\hat{a}_{i}}{b_{i}}\right)=\frac{1}{b_{i}^{2}}\left[P\left(\hat{\beta}_{i}\right)+P\left(\hat{\gamma}_{i}\right)\right]
$$

Finally, assuming that $\hat{\phi}_{i}$ is an unbiased estimate of $\phi_{i}$, a first-order Taylor series approximation of $\hat{\phi}_{i}$ is

$$
\hat{\phi}_{i} \approx \phi_{i}+\frac{\beta_{i}}{a_{i}^{2}}\left(\hat{\gamma}_{i}-\gamma_{i}\right)-\frac{\gamma_{i}}{a_{i}^{2}}\left(\hat{\beta}_{i}-\beta_{i}\right)
$$

Hence the variance of $\hat{\phi}_{i}$ is approximately given by

$$
P\left(\hat{\phi}_{i}\right) \approx\left[\frac{\beta_{i}^{2}}{a_{i}^{4}}\right] P\left(\hat{\gamma}_{i}\right)+\left[\frac{\gamma_{i}^{2}}{a_{i}^{4}}\right] P\left(\hat{\beta}_{i}\right)-2\left[\frac{\gamma_{i} \beta_{i}}{a_{i}^{4}}\right] \operatorname{cov}\left(\hat{\gamma}_{i}, \hat{\beta}_{i}\right)
$$

where, for simplicity, the estimated values are used in place of the true values. Note that, although the sinusoids will be orthogonal at integer multiples of the fundamental period, in general, they are not. Hence the covariance term in Eq. (14) must be included when computing the estimates at non-integer multiples of the fundamental period. For a discussion of the derivation of Eq. (14) along with the coefficients that should be used in practice since the true values of $\beta_{i}, \gamma_{i}$, and $a_{i}$ are unknown, refer to Appendix B.

\section{T-2 Subscale Jet Transport Aircraft}

The T-2 aircraft is a 5.5 percent dynamically scaled model of a generic commercial twin-engine jet transport aircraft. A photograph of the aircraft in flight is shown in Figure 4. The aircraft has twin jet engines mounted under the wings and retractable tricycle landing gear. Aircraft geometry and nominal mass properties are given in Table 1 below. Further information on the T-2 subscale jet aircraft and associated flight test operations can be found in Refs. [14]-[16].

\section{A. Control Surfaces}

Control surfaces on the aircraft are left and right ailerons, left and right inboard and outboard elevators, upper and lower rudders, left and right inboard and

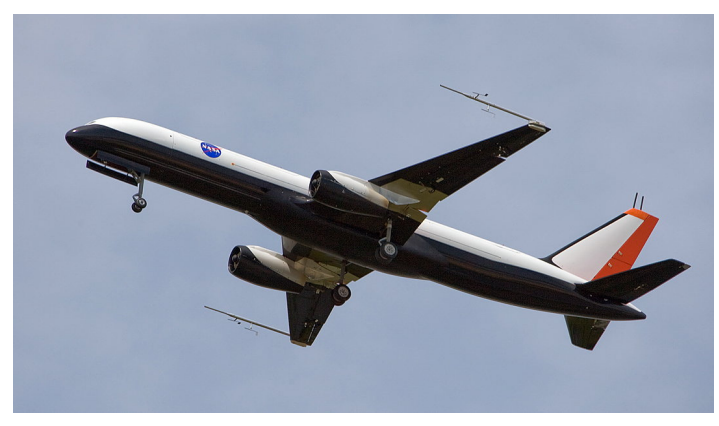

Figure 4. T-2 Subscale Jet Transport Aircraft Credit: NASA Langley Research Center outboard trailing-edge flaps, and left and right inboard and outboard spoilers, for a total of 16 independent control surfaces. For the data analyzed in this work, only the elevators, ailerons, and rudders were deflected. The individual elevator surfaces were moved together as a single elevator surface, and similarly for the rudders. Left and right ailerons were deflected asymmetrically, in the conventional way. Definitions of control surface deflections are 
given below. Trailing edge down is positive deflection for the wing and elevator surfaces, and trailing edge left is positive for the rudder.

$$
\begin{gathered}
\delta_{e}=\frac{1}{4}\left(\delta_{e_{\text {left outboard }}}+\delta_{e_{\text {left inboard }}}+\delta_{e_{\text {right outboard }}}+\delta_{e_{\text {right inboard }}}\right) \\
\delta_{a}=\frac{1}{2}\left(\delta_{a_{\text {right }}}-\delta_{a_{\text {left }}}\right) \quad \delta_{r}=\frac{1}{2}\left(\delta_{r_{\text {upper }}}+\delta_{r_{\text {lower }}}\right)
\end{gathered}
$$

The aircraft can be flown by a safety pilot using direct visual contact and conventional radio control. A research pilot executes flight test maneuvers from inside a mobile control room, using a synthetic vision display drawn from telemetry data and a local terrain database. Inputs from the research pilot and a ground-based flight control system are used to generate control surface commands which are transmitted by telemetry to the aircraft.

Table 1 T-2 Aircraft geometry and nominal mass properties

\begin{tabular}{|c|l|}
\hline $\bar{c}, \mathrm{ft}$ & 0.915 \\
\hline$b, \mathrm{ft}$ & 6.849 \\
\hline$S, \mathrm{ft}^{2}$ & 5.902 \\
\hline$x_{o}$, in & 57.30 \\
\hline$y_{o}$, in & 0.000 \\
\hline$z_{o}$, in & 11.28 \\
\hline$x_{c g}$, in & 56.63 \\
\hline$y_{c g}$, in & 0.000 \\
\hline$z_{c g}$, in & 11.43 \\
\hline$m$, slugs & 1.585 \\
\hline$I_{x}$, slugs- $\mathrm{ft}^{2}$ & 1.179 \\
\hline$I_{y}$, slugs- $\mathrm{ft}^{2}$ & 4.520 \\
\hline$I_{z}$, slugs- $\mathrm{ft}^{2}$ & 5.527 \\
\hline$I_{x z}$, slugs- $\mathrm{ft}^{2}$ & 0.211 \\
\hline \hline
\end{tabular}

\section{B. Instrumentation and Data Acquisition}

The T-2 aircraft was equipped with a micro-INS, which provided 3-axis translational accelerometer measurements, angular rate measurements, estimated attitude angles, and GPS velocity and position. Air data probes attached to booms mounted on each wingtip (visible in Figure 4) measured angle of attack, sideslip angle, static pressure, and dynamic pressure. Measurements from static pressure sensors and ambient temperature sensors were used to compute air density and altitude. Engine speeds in rpm were measured and used as inputs to an engine model to compute thrust. The engine model was identified from ground test data, with adjustments for ram drag identified from flight data. Potentiometers on the rotation axes of the control surfaces measured control surface deflections. Mass properties were computed based on measured fuel flow, pre-flight weight and balance, and inertia measurements of the aircraft on the ground. The pilot stick and rudder pedal commands and throttle position were also measured and recorded. Data from onboard sensors were telemetered to the ground in real time. Sampling rate for the flight data was $200 \mathrm{~Hz}$, decimated to $50 \mathrm{~Hz}$ for data analysis and modeling.

\section{Simulation Results}

A linearized longitudinal model for the T-2 subscale aircraft driven by an optimized multi-sine was used for simulation testing. The measured outputs were corrupted with colored noise representative of both sensor noise and nonlinearities not captured by the linear model. The recursive frequency response estimation algorithm given previously was tested to demonstrate the accuracy of the estimates and their 95 percent confidence intervals $(2 \sigma$ uncertainty bounds) after one and two periods of the input signal. 
The linearized longitudinal model for the T-2 subscale aircraft was

$$
\left[\begin{array}{l}
\dot{\alpha}(t) \\
\dot{q}(t)
\end{array}\right]=\left[\begin{array}{ll}
-2.4475 & 0.99709 \\
-34.896 & -3.8467
\end{array}\right]\left[\begin{array}{l}
\alpha(t) \\
q(t)
\end{array}\right]+\left[\begin{array}{c}
-0.18174 \\
-39.963
\end{array}\right] \delta_{e}(t)
$$

where $\alpha$ denotes the angle of attack in radians, $q$ denotes the body-axis pitch rate in radians per second, and $\delta_{e}$ denotes the elevator deflection in radians. The output model was

$$
y(t)=\left[\begin{array}{c}
\alpha(t) \\
q(t) \\
a_{z}(t)
\end{array}\right]=\left[\begin{array}{cc}
1 & 0 \\
0 & 1 \\
-9.8318 & -0.011702
\end{array}\right]\left[\begin{array}{l}
\alpha(t) \\
q(t)
\end{array}\right]+\left[\begin{array}{c}
0 \\
0 \\
-0.73005
\end{array}\right] \delta_{e}(t)
$$

where $a_{z}$ denotes the $z$ body-axis translational acceleration in $\mathrm{g}$.

The elevator deflection $\delta_{e}$ was chosen to be an optimized multi-sine ${ }^{11}$ with frequency content spaced uniformly at $0.1 \mathrm{~Hz}$ intervals between $0.1 \mathrm{~Hz}$ and $2.6 \mathrm{~Hz}$. Figure 5 shows the input and states of the longitudinal aircraft model over one period of the input, which was 10 seconds.

Let $z(t)$ denote a measurement of $y(t)$ which is corrupted by additive zero-mean colored measurement noise,

$$
z(t)=y(t)+v(t)
$$

where $z$ has a signal-to-noise ratio of 10 , and $v$ is the sum of a $2 \mathrm{~Hz}$ band-limited noise component and a wide-band noise component of equal power. As an indication of the type of noise in the output measurements, Figure 6 displays the power-spectral density of the $2 \mathrm{~Hz}$ band-limited noise component in the measurement of $\alpha$. Note that this type of noise is representative of model residuals seen in practice due to nonlinearities not captured by Eqs. (15)-(16).

Applying the recursive estimation algorithm presented previously, Figures 7, 8, and 9 show comparisons of the true and estimated Bode plots for all three measured outputs after 1 and 2 periods of the input. The estimated $2 \sigma$ error bounds are displayed when 11 terms are used in the estimated autocorrelation, that is, $s=10$. The figures show that uncertainty in the phase is large at points for which the frequency response amplitude is small. This is because the variance in the estimated phase is inversely proportional to $a_{i}^{4}$, cf. Eq. (14). For an intuitive description of this phenomenon, see Appendix C. For several frequency points, after 1 period of the input, the true frequency response functions were not within the estimated $2 \sigma$ error bounds. However, after 2 periods of the input, the estimated $2 \sigma$ error bounds tend to include the true frequency response functions.

In this work, $s$ was taken to be 10 because the complete autocorrelation function indicated that the autocorrelation function was negligible after the tenth term. However, when estimating the autocorrelation function recursively, this approach is not possible, in which case $s$ could be chosen from experience. The choice of $s$ is a balance between accuracy and computational burden. Taking $s$ to be equal to the number of data points would allow characterization of the most general noise autocorrelation functions, although this choice would not be amenable to recursive computation. Conversely, taking $s$ small, such that some significant terms in the noise autocorrelation function would not be included, would lead to poor estimates of the covariance. As an alternative, it might be possible to model the autocorrelation function accurately with only a few terms by recursively estimating a parametric model for the autocorrelation function, if a general form for the autocorrelation function was known. 

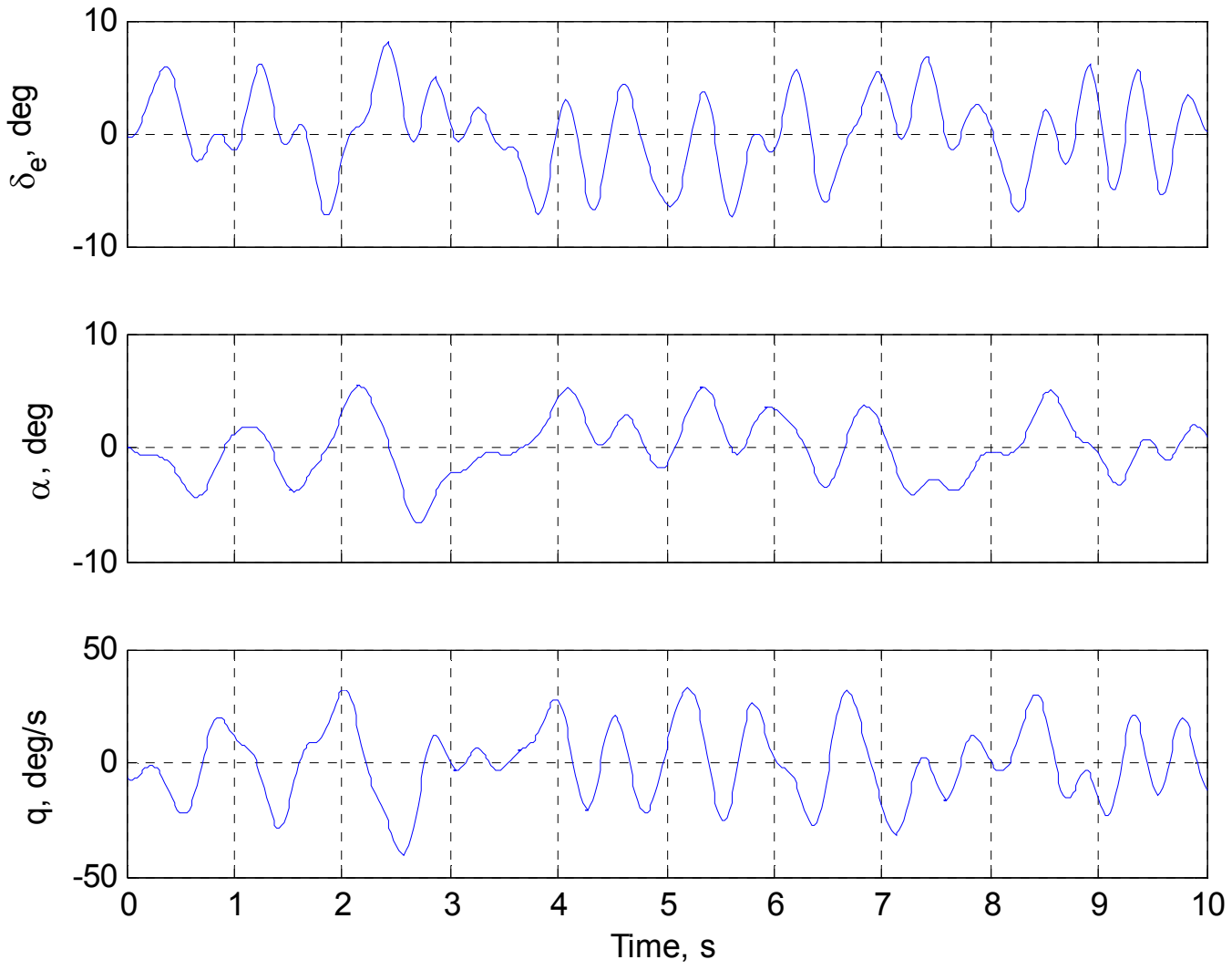

Figure 5. Input and outputs for the longitudinal aircraft model over one period of the input

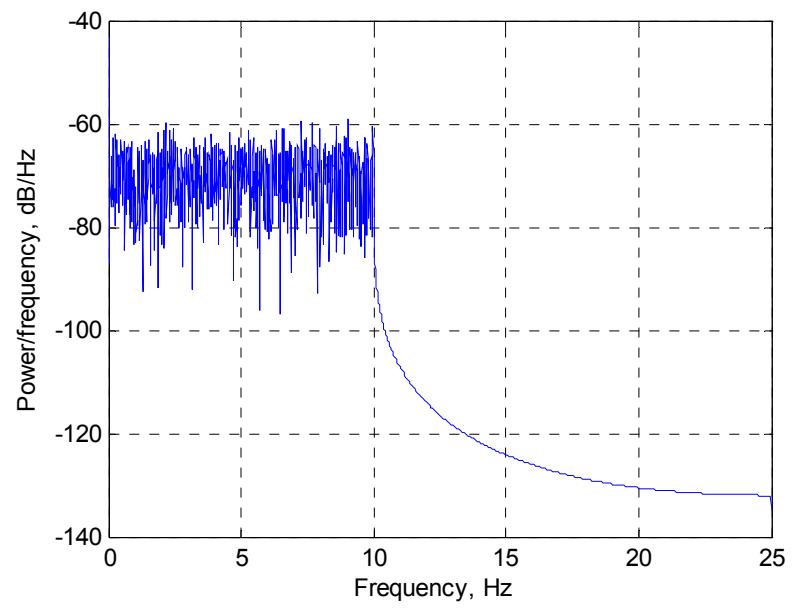

Figure 6. Power-spectral density of the $2 \mathrm{~Hz}$ band-limited noise component in the measurement of $\alpha$ 

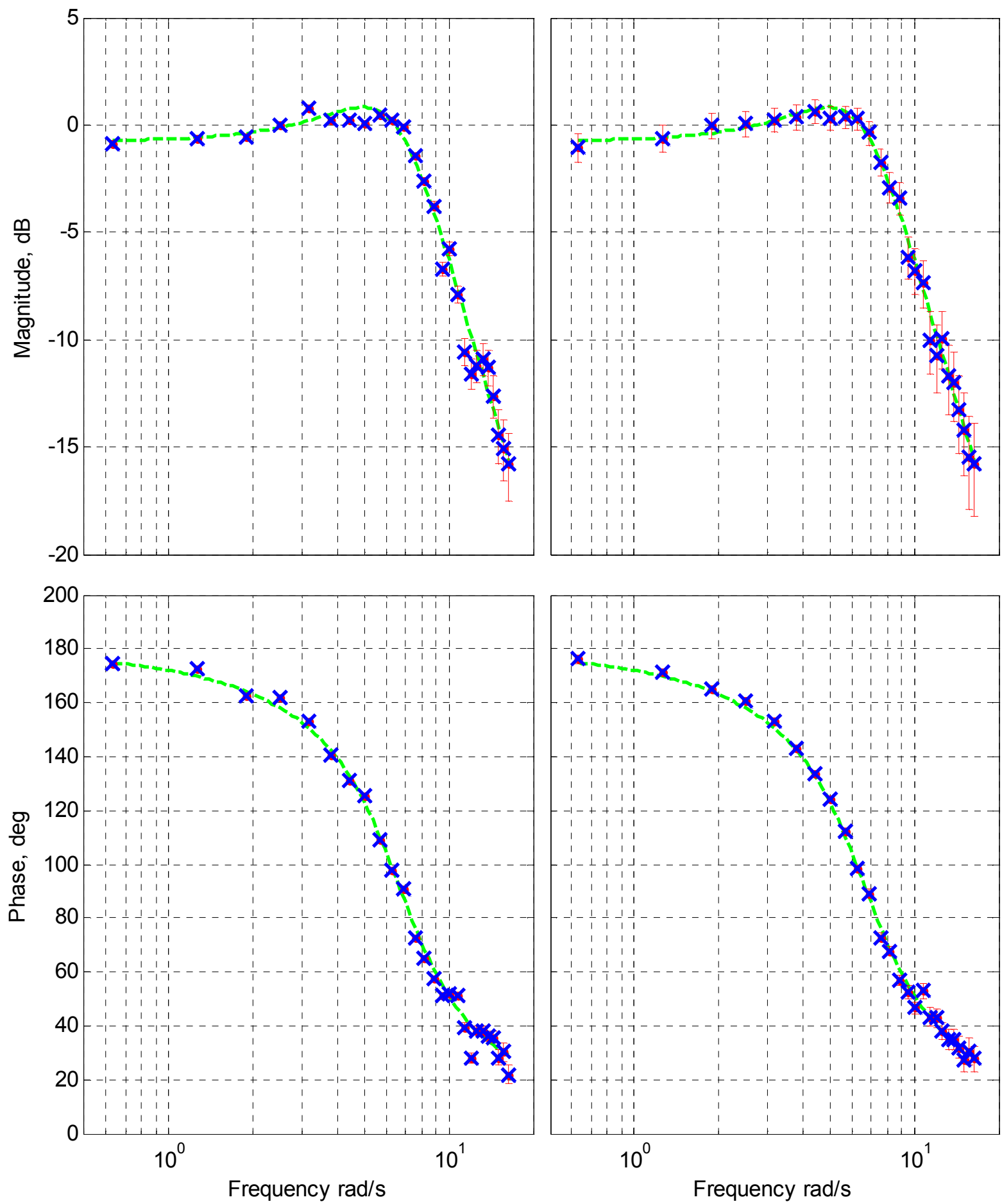

Figure 7. Comparison of the true ('--') and estimated ('x') Bode plots from $\delta_{e} \rightarrow \alpha$ after 1 (left) and 2 (right) periods of the input; error bars represent the estimated $2 \sigma$ error bounds when $s=10$ 

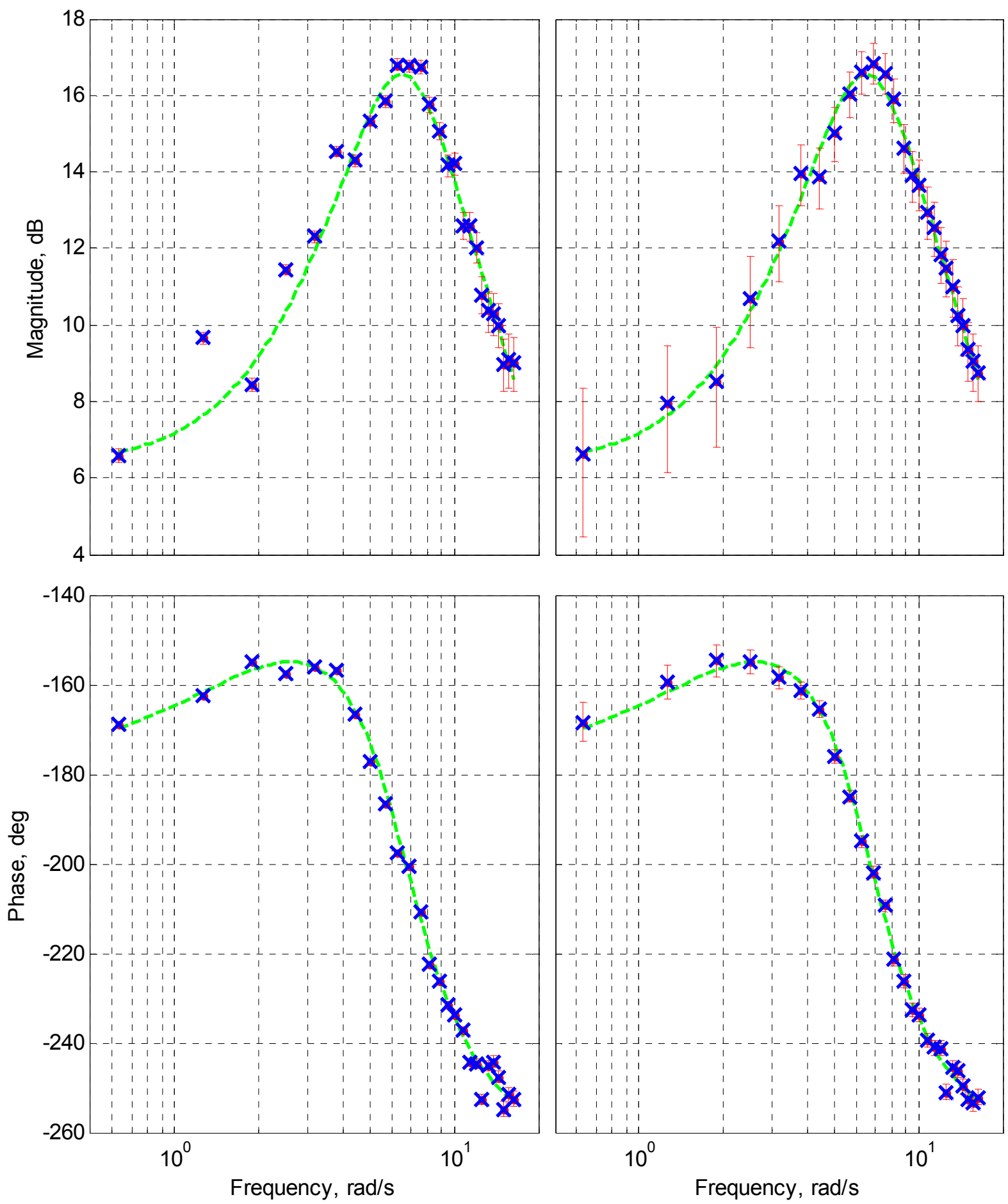

Figure 8. Comparison of the true ('--') and estimated ('x') Bode plots from $\delta_{e} \rightarrow q$ after 1 (left) and 2 (right) periods of the input; error bars represent the estimated $2 \sigma$ error bounds when $s=10$ 

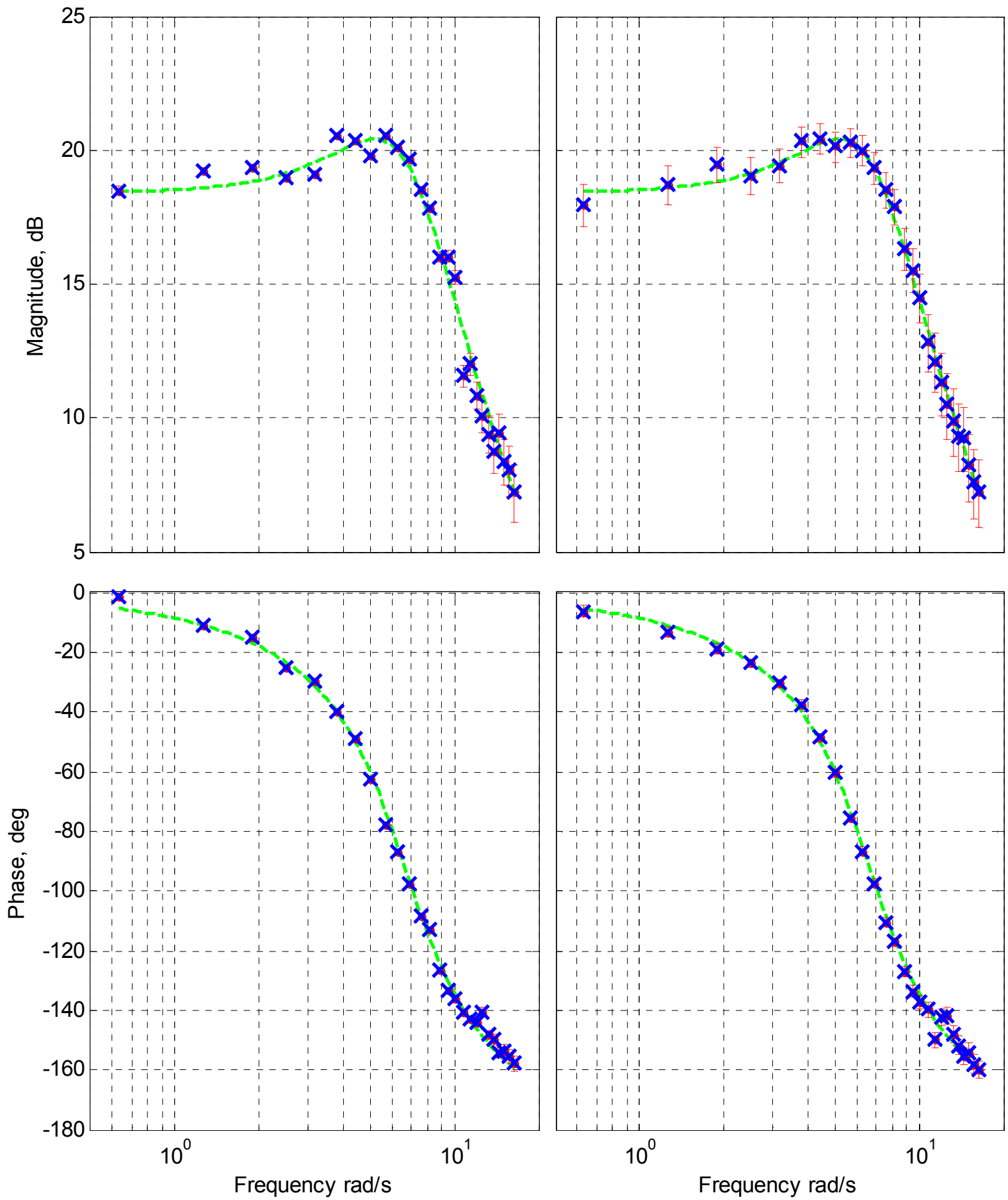

Figure 9. Comparison of the true ('--') and estimated ('x') Bode plots from $\delta_{e} \rightarrow a_{z}$ after 1 (left) and 2 (right) periods of the input; error bars represent the estimated $2 \sigma$ error bounds when $s=10$ 


\section{Flight Results}

Frequency responses for the T-2 aircraft from the elevator deflection $\delta_{e}$ to angle of attack $\alpha$, body-axis pitch rate $q$, and $z$ body-axis translational acceleration $a_{z}$ were estimated recursively, in the manner described previously. Flight data were gathered from a 10 -second multi-axis maneuver, in which the elevators, ailerons, and rudders were excited with phase-optimized mutually orthogonal multi-sines ${ }^{11}$. The elevator, aileron, and rudder perturbation inputs were chosen to be multi-sines with frequency content uniformly-spaced at $0.3 \mathrm{~Hz}$, where the elevator had frequency content from 0.3 to $2.1 \mathrm{~Hz}$, the aileron had frequency content from 0.4 to $2.2 \mathrm{~Hz}$, and the rudder had frequency content from 0.2 to $2.0 \mathrm{~Hz}$; hence none of the command inputs had overlapping frequency content. Note that the frequency content in each of the inputs was sparser than in the simulation case shown earlier, because the time length of the flight maneuver was necessarily short due to test range constraints, and because the frequency content was distributed among the three control inputs.

In the recursive estimation algorithm, as a preliminary step towards obtaining the frequency responses, estimates of the magnitude and phase of the sinusoidal components of a signal are computed, allowing one to construct an approximate signal. Assuming frequency content at $0 \mathrm{~Hz}$ (to account for any bias) and 0.3 to $2.1 \mathrm{~Hz}$ at $0.3 \mathrm{~Hz}$ intervals for the measured signals $\delta_{e}, \alpha, q$, and $a_{z}$, Figure 10 shows a comparison of the measured and estimated signals. Note that none of the measured signals is exactly represented as a sum of sines, even the input signal $\delta_{e}$. These small inaccuracies are the result of actuator dynamics and nonlinearities, as well as nonlinear effects in the aircraft dynamics and aerodynamics. Variance estimates for the frequency responses will not incorporate the effects of these small inaccuracies. Most of the mismatch is around the peaks of the waveforms, which is characteristic of nonlinear effects.

Applying the recursive estimation algorithm presented previously, Figures 11, 12, and 13 compare the estimated frequency response Bode plots after 4 and 10 seconds for all three measured outputs to the Bode plots generated from the linearized longitudinal model presented previously in Eqs. (15) and (16). The linear model of Eqs. (15) and (16) was identified from the same flight data using conventional output-error parameter estimation in the time domain ${ }^{11,12}$. Estimated $2 \sigma$ error bounds are displayed for 11 terms used in the estimated autocorrelation, that is, $s=10$. From Figures 11, 12, and 13, it appears that the linearized longitudinal model is in good agreement with the frequency response magnitudes estimated in real time, although the frequency response phases appear to be slightly biased. 

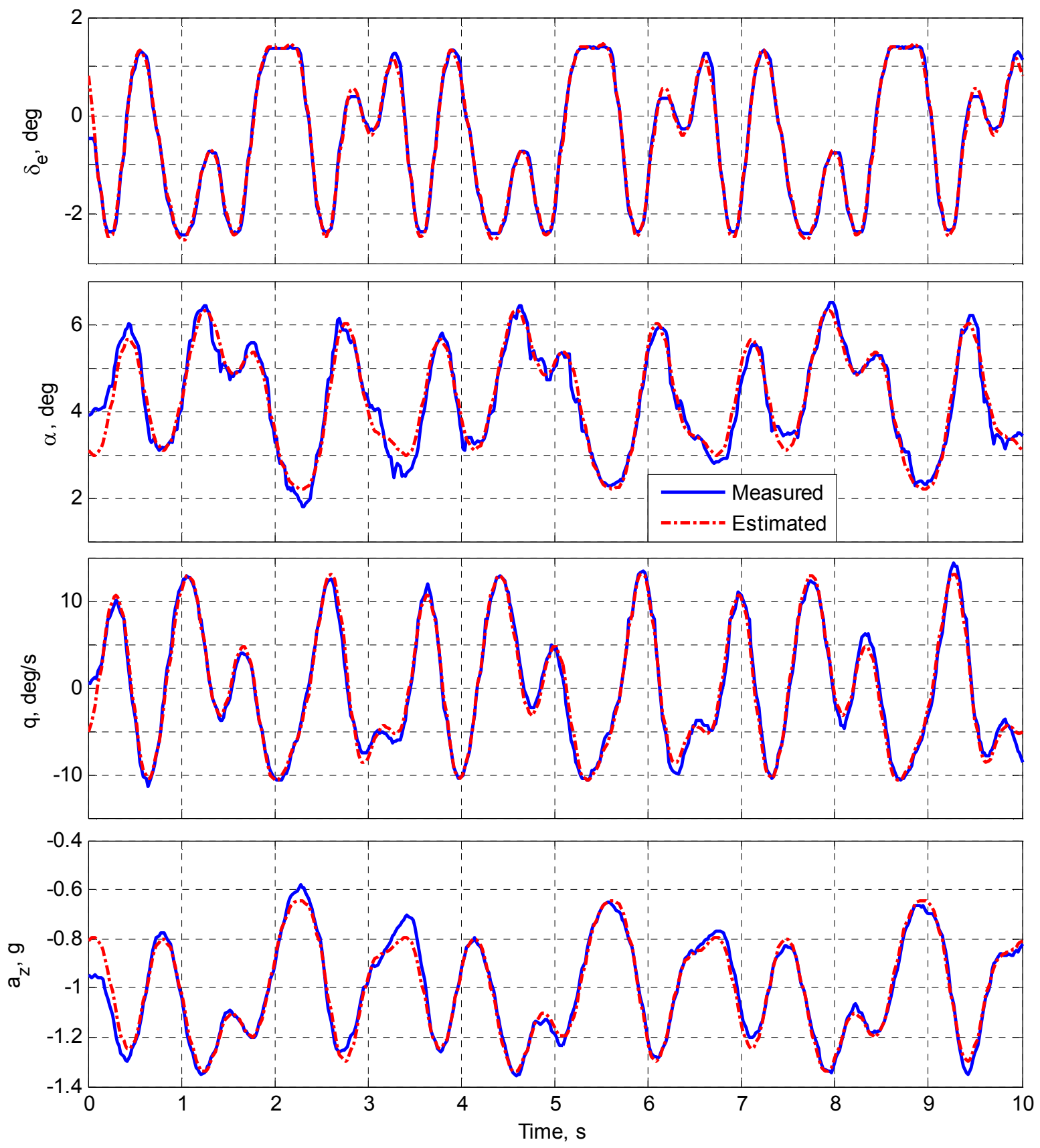

Figure 10. Measured and Fourier approximation of elevator deflection, angle of attack, body-axis pitch rate, and $\mathrm{z}$ body-axis translational acceleration 

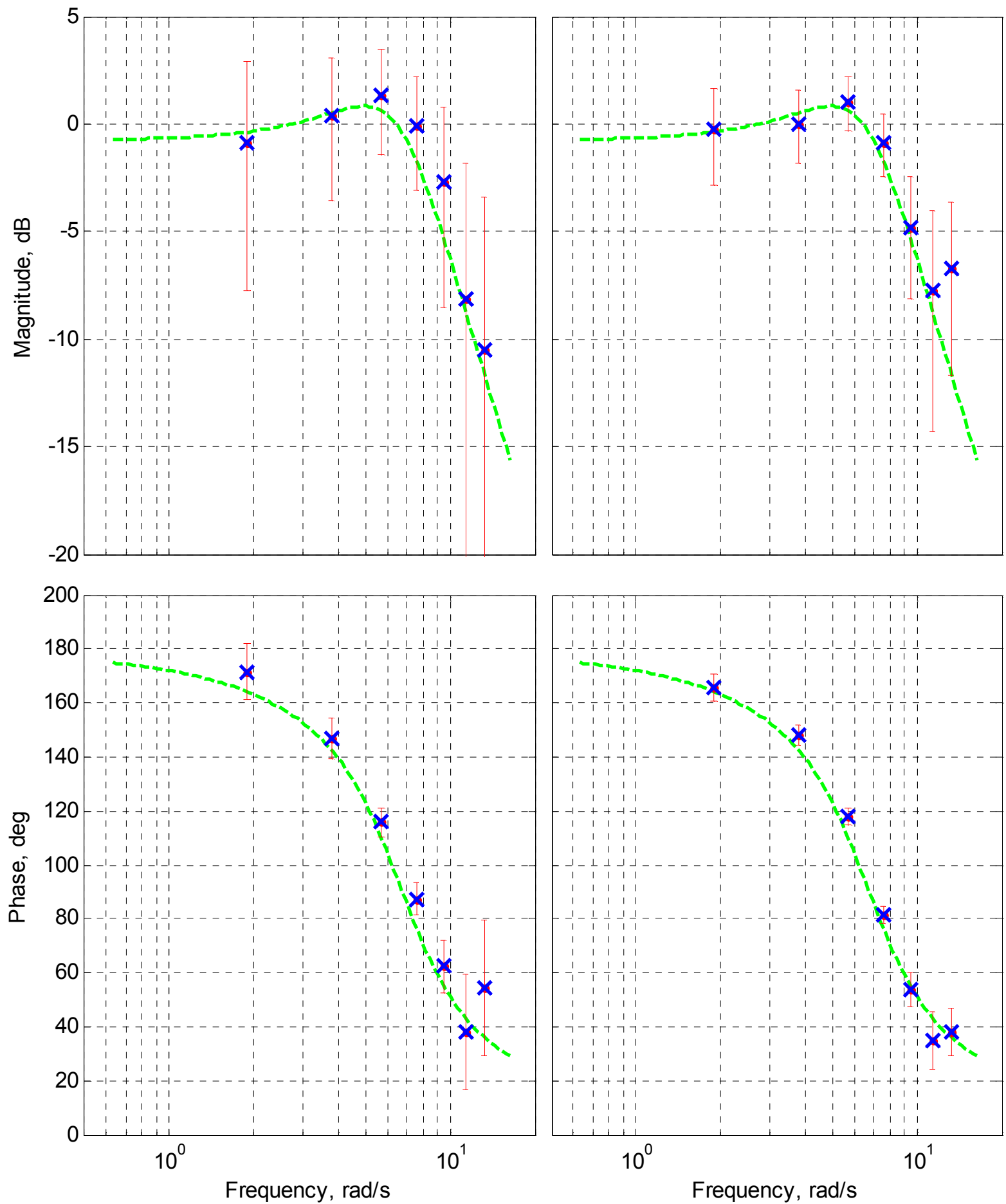

Figure 11. Comparison of the linear ('--') and estimated ('x') Bode plots from $\delta_{e} \rightarrow \alpha$ after 4s (left) and 10s (right); error bars represent the estimated $2 \sigma$ error bounds when $s=10$ 

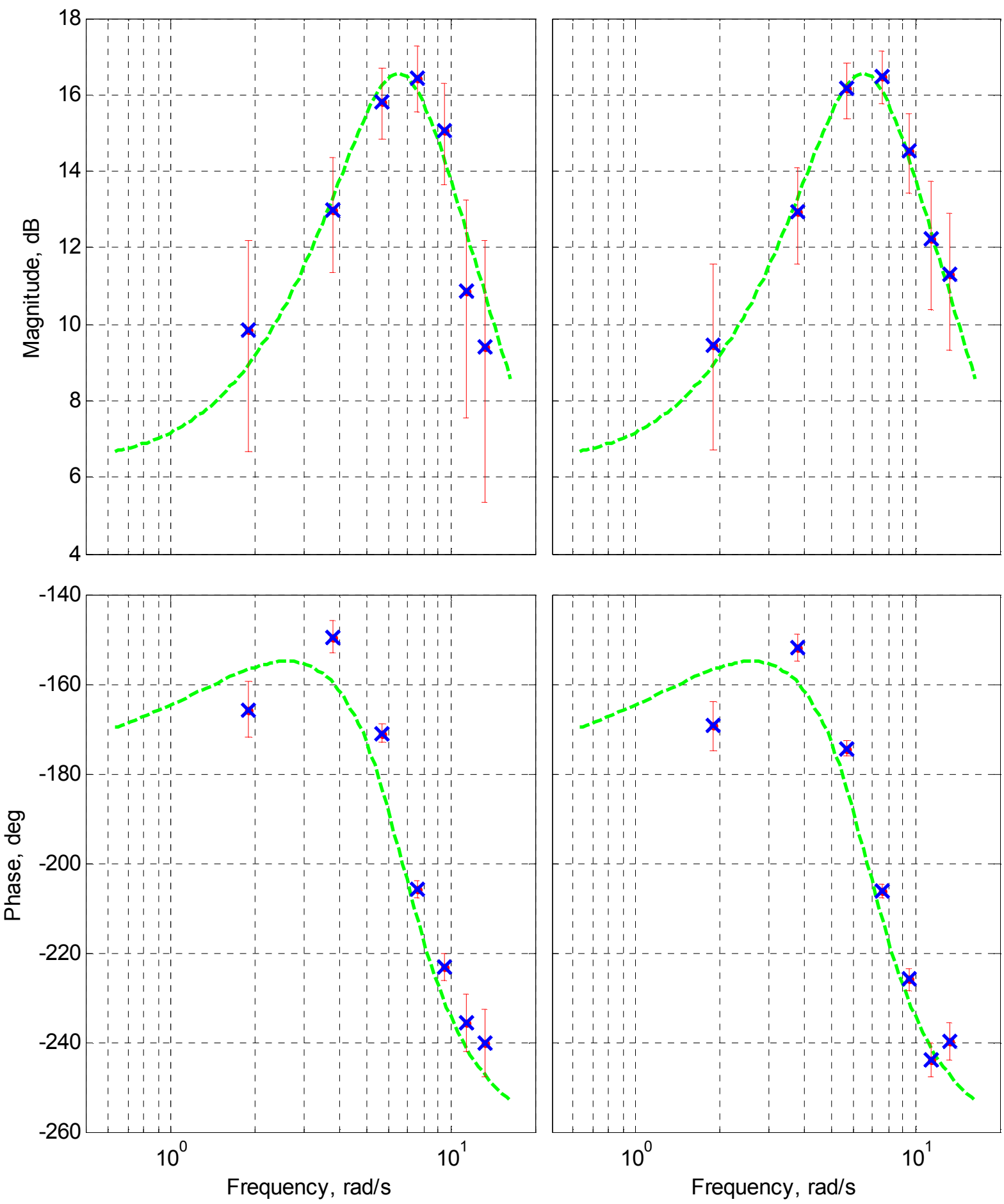

Figure 12. Comparison of the linear ('--') and estimated (' $x$ ') Bode plots from $\delta_{e} \rightarrow q$ after $4 \mathrm{~s}$ (left) and 10s (right); error bars represent the estimated $2 \sigma$ error bounds when $s=10$ 

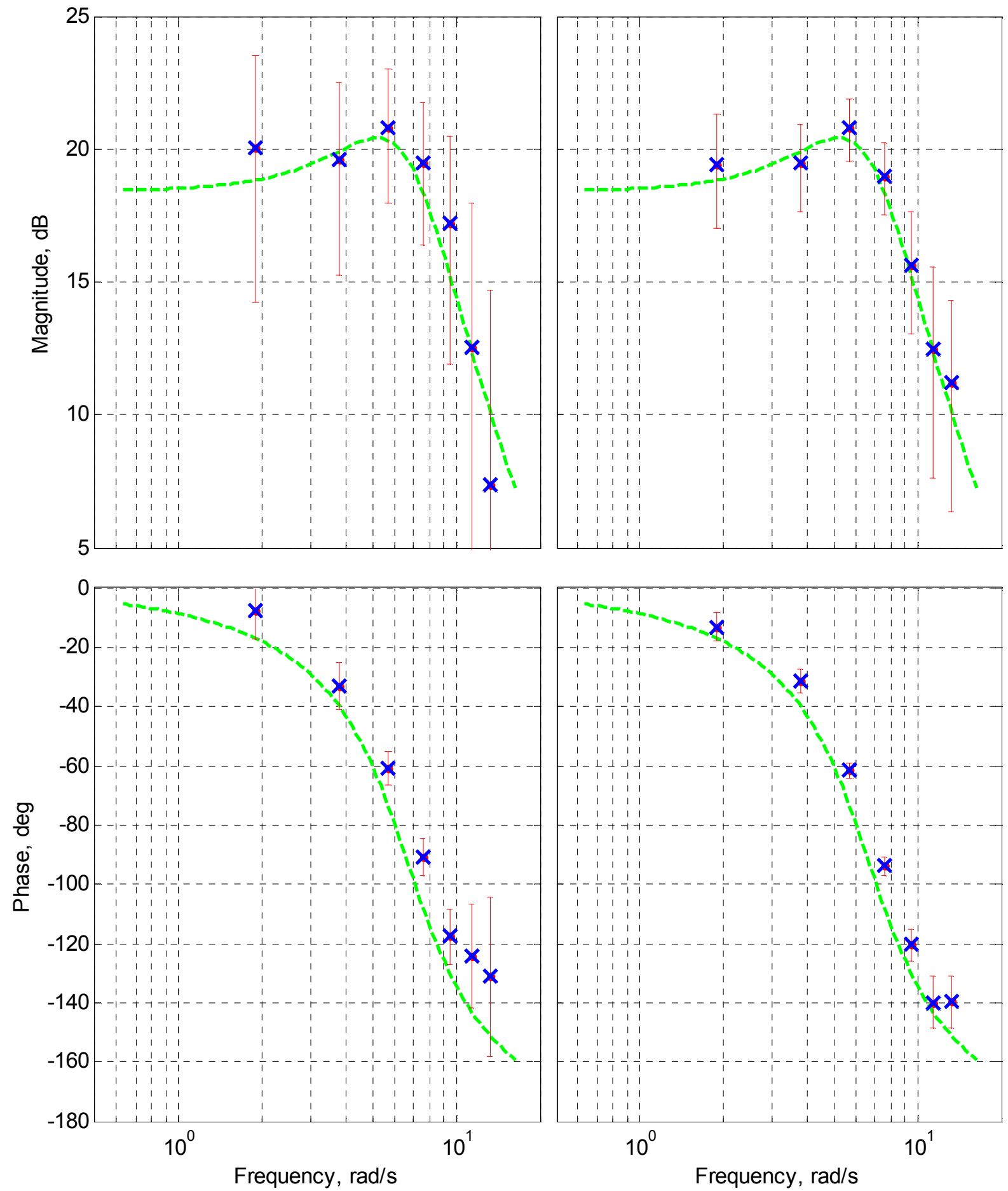

Figure 13. Comparison of the linear ('--') and estimated ('x') Bode plots from $\delta_{e} \rightarrow a_{z}$ after 4s (left) and 10 s (right); error bars represent the estimated $2 \sigma$ error bounds when $s=10$ 


\section{Error Sources}

Numerous sources of error arise when implementing the aforementioned algorithm for recursive frequency response estimation, which are pointed out here to aid the practitioner in diagnosing any problems that may arise. All or some of these factors may have contributed to inaccuracies in the previous section.

First, the present paper focuses on the use of the Fourier transform for estimating the frequency response. Specifically, the frequency response is taken to be the ratio of the Fourier transform of the output to the Fourier transform of the input. However, this method of computing the frequency response is technically only valid when the output is in steady-state, that is, when the initial condition response of the system has died out. For measurements of an aircraft in flight, this is almost never exactly the case, which may account for some of the errors in the previous section. The adverse impact of initial conditions is common in nonparametric identification, and typically the methods used to address this problem are to either wait for the initial condition response to die out, or to employ a parametric model structure, which may be difficult to ascertain.

Second, the present paper assumes that the system is approximately linear. However, nonlinearities can contribute frequency content in the output at frequencies that were not present in the input. For instance, a classical example in the literature is when the output is a cubic function of the input, that is,

$$
y(t)=[u(t)]^{3}
$$

Then letting $u(t)=a \cos (\omega t+\phi)$, it follows that

$$
y(t)=\frac{3}{4} a^{3} \cos (\omega t+\phi)+\frac{1}{4} a^{3} \cos (3 \omega t+3 \phi)
$$

Hence a cubic nonlinearity contributes frequency content in the output at the input frequency and 3 times the input frequency. Similar expressions can be derived easily for most polynomial nonlinearities.

Third, the present paper assumes that the autocorrelation function of the noise only has a finite number of terms, an upper bound of which should be known a priori. This assumption may break down in the presence of deterministic modeling errors, such as unmodeled frequency content.

Fourth, the estimates of the covariance bounds are themselves based on the estimates of the Fourier transform. Poor estimates can therefore lead to poor error bounds. Furthermore, the present paper assumes that the estimates of the magnitude and phase are unbiased when determining the variance of the frequency response, although whether or not this is a realistic assumption depends strongly on the noise properties of a given problem. Also, when computing the variances of the phase estimates, linearization was employed, so that the accuracy of the phase error bound is dependent on how close the estimated phase is to the true phase.

Finally, the properties of the residual sequences will determine how quickly the estimates of the autocorrelation function converge. For finite data, there is no guarantee on the accuracy of the estimated autocorrelation function, and this impacts the accuracy of the estimated parameter covariances.

\section{Concluding Remarks}

A method for estimating frequency responses in real time was developed and demonstrated. The method uses recursive least squares with sinusoidal regressors, in conjunction with a known optimized multi-sine input design. The problem was formulated in a novel way, and solved using conventional recursive least squares and an accurate method for recursive calculation of the model parameter covariance matrix. The recursive method for covariance matrix estimation in the time domain, described here for the first time, can account for colored residuals typically encountered in practical aircraft dynamic modeling problems. Results from simulation testing and flight data analysis showed that the approach can be used to achieve accurate estimates of frequency responses in real time using short data records. Because frequency response estimates are a nonparametric characterization of dynamic systems, this capability has very general applications to tasks such as efficient flight testing for aircraft flight envelope expansion, as well as real-time dynamic modeling required by adaptive or reconfigurable control algorithms for nominal or damaged aircraft.

\section{Acknowledgements}

The efforts of the AirSTAR flight test team at NASA Langley, in building and testing the aircraft and associated systems, carefully calibrating the instrumentation, and carrying out the flight operations to collect the high-quality flight data used in this study, are gratefully acknowledged. Research in dynamic modeling is funded by the NASA Aviation Safety Program, Integrated Resilient Aircraft Control (IRAC) project. The generous funding of the NASA Aeronautics Scholarship Program, through which this collaborative effort was made possible, is also acknowledged. 


\section{References}

${ }^{1}$ Bosworth, J.T. and West, J.C., "Real-Time Open-Loop Frequency Response Analysis of Flight Test Data," AIAA-86-9738, AIAA/AHS/CASl/DGLR/IES/ISIB/ITEA/SETP/SFTE 3rd Flight Testing Conference, Las Vegas, NV, April 1986.

${ }^{2}$ Bosworth, J. T. and Burken, J.J. "Tailored Excitation for Multivariable Stability-Margin Measurement Applied to the X-31A Nonlinear Simulation," NASA TM-113085, 1997.

${ }^{3}$ Lisoski, D.L. and Tischler, M.B. "Solar Powered Stratospheric Research Aircraft - Flight Test and System Identification," Paper 27, RTO SCI Symposium on System Identification for Integrated Aircraft Development and Flight Testing, RTO MP-11, Madrid, Spain, May 1998.

${ }^{4}$ Balough, D. L., "Determination of X-36 Stability Margins Using Real-Time Frequency Response Techniques," AIAA-98-4154, August 1998

${ }^{5}$ Colin T., Tischler, M., and Colbourne, J. "Rapid Frequency-Domain Modeling Methods for Unmanned Aerial Vehicle Flight Control Applications," Journal of Aircraft, Vol.41, No.4, 2004, pp. 735-743.

${ }^{6}$ Bosworth, J.T. and Stachowiak, S.J. "Real-Time Stability Margin Measurements for X-38 Robustness Analysis," NASA/TP-2005-212856, February 2005.

${ }^{7}$ Regan, C. "In-Flight Stability Analysis of the X-48B Aircraft," AIAA-2008-6571, AIAA Atmospheric Flight Mechanics Conference and Exhibit, Honolulu, HI, August 2008

${ }^{8}$ Juang, Jer-Nan Applied System Identification, Prentice-Hall PTR, Upper Saddle River, NJ, 1994.

${ }^{9}$ Chen, C., Lee, G., and Juang, J. "Several Recursive Techniques for Observer/Kalman Filter System Identification from Data," AIAA-92-4386, AIAA Navigation, Guidance, and Control Conference, Hilton Head, SC, August 1992.

${ }^{10}$ Peyton Jones, J.C. and Billings, S.A. "Recursive Algorithm for Computing the Frequency Response of a Class of Nonlinear Difference Equation Models," International Journal of Control, Vol. 50, No. 5, November 1989, pp. 1924-1940.

${ }^{11}$ Klein, V. and Morelli, E.A. Aircraft System Identification - Theory and Practice, AIAA Education Series, AIAA, Reston, VA, 2006.

${ }^{12}$ Morelli, E.A. and Klein, V., "Accuracy of Aerodynamic Model Parameters Estimated from Flight Test Data," Journal of Guidance, Control, and Dynamics, Vol. 20, No. 1, January-February 1997, pp. 74-80.

${ }^{13}$ Schroeder, M.R. "Synthesis of Low-Peak-Factor Signals and Binary Sequences with Low Autocorrelation," IEEE Transactions on Information Theory, Vol. 16, No. 1, 1970, pp. 85-89.

${ }^{14}$ Jordan, Thomas L., Langford, William M., and Hill, Jeffrey S.; "Airborne Subscale Transport Aircraft Research Testbed: Aircraft Model Development", AIAA 2005-6432, AIAA Guidance, Navigation, and Control Conference and Exhibit, August 2005.

${ }^{15}$ Jordan, Thomas L., Foster, John V., Bailey, Roger M., and Belcastro, Christine M., "AirSTAR: A UAV Platform for Flight Dynamics and Control System Testing," AIAA-2006-3307, 25th AIAA Aerodynamic Measurement Technology and Ground Testing Conference, San Francisco, CA, June 2006.

${ }^{16}$ Murch, A. M., "A Flight Control System Architecture for the NASA AirSTAR Flight Test Facility," AIAA 2008-6990, AIAA Guidance, Navigation, and Control Conference and Exhibit, Honolulu, HI, August 2008. 


\section{Appendix}

\section{A. The Vec Operator and the Kronecker Product}

Let $A, B$, and $C$ be matrices, where $A$ is given by

$$
A=\left[\begin{array}{ccc}
a_{1,1} & \ldots & a_{1, n} \\
\vdots & & \vdots \\
a_{l, 1} & \ldots & a_{l, n}
\end{array}\right]
$$

and $B$ and $C$ are of arbitrary dimensions. Then $\operatorname{vec}(A)$ is a vector made by stacking the columns of $A$, that is, letting $\vec{a}_{i}$ denote the $i^{\text {th }}$ column of $A$,

$$
\operatorname{vec}(A)=\left[\begin{array}{lll}
\vec{a}_{1}^{T} & \cdots & \vec{a}_{n}^{T}
\end{array}\right]^{T}
$$

The Kronecker product of $A$ and $B$, denoted $A \otimes B$, is given by

$$
A \otimes B=\left[\begin{array}{ccc}
a_{1,1} B & \ldots & a_{1, n} B \\
\vdots & & \vdots \\
a_{l, 1} B & \ldots & a_{l, n} B
\end{array}\right]
$$

where, in general, $A \otimes B \neq B \otimes A$.

Throughout the paper, the following identity is employed

$$
\operatorname{vec}(B A C)=\left(C^{T} \otimes B\right) \operatorname{vec}(A)
$$

where $B, A$, and $C$ have conformable dimensions for multiplication.

\section{B. Derivation of the Phase Uncertainty and Discussion of Implementable Coefficients}

As previously assumed in the derivation of Eq. (14), assume that $\hat{\phi}_{i}$ is an unbiased estimate of $\phi_{i}$, that is,

$$
\mathrm{E}\left[\hat{\phi}_{i}\right]=\mathrm{E}\left[\tan ^{-1}\left(\frac{\hat{\beta}_{i}}{\hat{\gamma}_{i}}\right)\right]=\phi_{i}
$$

Then from the definition of $\hat{\phi}_{i}$, a first-order Taylor series approximation of $\hat{\phi}_{i}$ is given by

$$
\hat{\phi}_{i} \approx \phi_{i}+\frac{\beta_{i}}{a_{i}^{2}}\left(\hat{\gamma}_{i}-\gamma_{i}\right)-\frac{\gamma_{i}}{a_{i}^{2}}\left(\hat{\beta}_{i}-\beta_{i}\right)
$$

Hence the variance of $\hat{\phi}_{i}$ is approximately given by

$$
\begin{gathered}
P\left(\hat{\phi}_{i}\right)=\mathrm{E}\left[\left(\hat{\phi}_{i}-\phi_{i}\right)^{2}\right] \\
=\mathrm{E}\left[\frac{\beta_{i}}{a_{i}^{2}}\left(\hat{\gamma}_{i}-\gamma_{i}\right)-\frac{\gamma_{i}}{a_{i}^{2}}\left(\hat{\beta}_{i}-\beta_{i}\right)\right]^{2} \\
=\left[\frac{\beta_{i}^{2}}{a_{i}^{4}}\right] \mathrm{E}\left[\left(\hat{\gamma}_{i}-\gamma_{i}\right)^{2}\right]+\left[\frac{\gamma_{i}^{2}}{a_{i}^{4}}\right] \mathrm{E}\left[\left(\hat{\beta}_{i}-\beta_{i}\right)^{2}\right]-2\left[\frac{\gamma_{i} \beta_{i}}{a_{i}^{4}}\right] \mathrm{E}\left[\left(\hat{\gamma}_{i}-\gamma_{i}\right)\left(\hat{\beta}_{i}-\beta_{i}\right)\right]
\end{gathered}
$$

or, equivalently, Eq. (14).

Next, note that even though $\beta_{i}, \gamma_{i}, a_{i}$, the variance of $\hat{\gamma}_{i}$, the variance of $\hat{\beta}_{i}$, and the covariance of $\hat{\gamma}_{i}$ and $\hat{\beta}_{i}$ can be estimated, plugging all of the estimated values into Eq. (14) may not yield an unbiased estimate of the phase variance. For instance, the covariance of $\hat{\beta}_{i}^{2}$ is equivalently given by

$$
\begin{aligned}
P\left(\hat{\beta}_{i}\right) & =\mathrm{E}\left[\hat{\beta}_{i}^{2}\right]-\mathrm{E}\left[\hat{\beta}_{i}\right]^{2} \\
& =\mathrm{E}\left[\hat{\beta}_{i}^{2}\right]-\beta_{i}^{2}
\end{aligned}
$$

Hence an unbiased estimate of $\beta_{i}^{2}$ is given by

$$
\beta_{i}^{2}=\hat{\beta}_{i}^{2}-P\left(\hat{\beta}_{i}\right)
$$

since $E\left[\hat{\beta}_{i}^{2}-P\left(\hat{\beta}_{i}\right)\right]=\beta_{i}^{2}$. Similarly, unbiased estimates of $\gamma_{i}^{2}$ and $\beta_{i} \gamma_{i}$ are given by 


$$
\begin{aligned}
\gamma_{i}^{2} & =\hat{\gamma}_{i}^{2}-P\left(\hat{\gamma}_{i}\right) \\
\gamma_{i} \beta_{i} & =\hat{\gamma}_{i} \hat{\beta}_{i}-\operatorname{cov}\left(\hat{\gamma}_{i}, \hat{\beta}_{i}\right)
\end{aligned}
$$

where it is important to note that $\hat{\beta}_{i}, \hat{\gamma}_{i}, P\left(\hat{\beta}_{i}\right), P\left(\hat{\gamma}_{i}\right)$, and $\operatorname{cov}\left(\hat{\gamma}_{i}, \hat{\beta}_{i}\right)$ can be estimated from the data. However, to calculate an unbiased estimate of $a_{1}^{4}$ would require knowledge of the higher statistics of $\hat{\beta}_{i}$ and $\hat{\gamma}_{i}$. Hence if $\hat{a}_{i}^{4}$ was used in Eq. (14) in place of $a_{1}^{4}$, but all of the other values were replaced with their unbiased estimates, the estimated covariance in the phase would be smaller than the true value. Therefore, the estimated values are simply used in place of their true values in Eq. (14), even though this may yield a biased estimate of the phase variance.

\section{Phase Uncertainty}

In this work, the magnitude and phase of the Fourier transform are estimated indirectly. Specifically, $\beta_{i} \triangleq$ $a_{i} \cos \left(\phi_{i}\right)$ and $\gamma_{i} \triangleq a_{i} \sin \left(\phi_{i}\right)$ are estimated instead of $a_{i}$ and $\phi_{i}$ directly, where the relationship between $\beta_{i}, \gamma_{i}, a_{i}$, and $\phi_{i}$ is graphically depicted in the following figure.

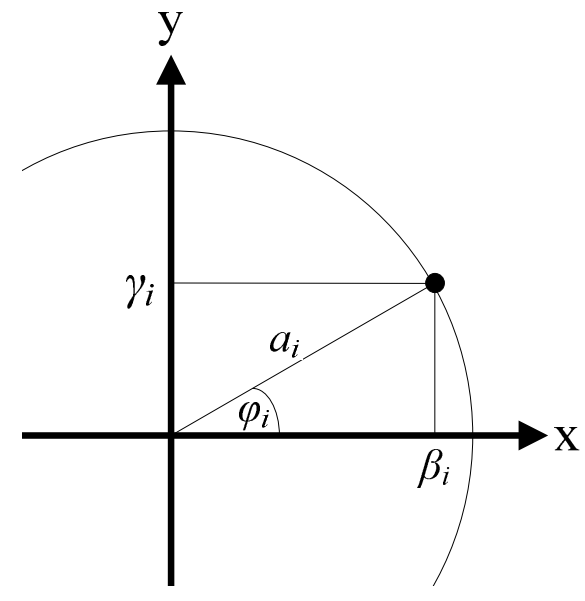

When there is uncertainty in the estimates of $\beta_{i}$ and $\gamma_{i}$, denoted by $\hat{\beta}_{i}$ and $\hat{\gamma}_{i}$, the uncertainty in $\widehat{\phi}_{i}$ is very dependent on the magnitude $a_{i}$. Specifically, from Eq. (14), the variance of $\hat{\phi}_{i}$ is inversely proportional to $a_{i}^{4}$. An intuitive understanding for this dependence can be based on the following example.

Example 3 Let the estimates $\hat{\beta}_{i}$ and $\hat{\gamma}_{i}$ be such that the point $\mathbf{z}=\beta_{i} \mathbf{x}+\gamma_{i} \mathbf{y}$ will lie in some circular region in the XY plane about the point $\hat{\mathbf{z}}=\hat{\beta}_{i} \mathbf{x}+\hat{\gamma}_{i} \mathbf{y}$. Specifically, consider two cases. In the first case, the point $\hat{\mathbf{z}}$ will have a phase of $\hat{\phi}$ and magnitude of $\hat{a}$, and $\mathbf{z}$ will lie in a circle of radius $r$ about $\hat{\mathbf{z}}$. In the second case, the point $\hat{\mathbf{z}}$ will have a phase of $\hat{\phi}$ and magnitude of $\hat{a} / 10$, and $\mathbf{z}$ will lie in a circle of radius $r$ about $\hat{\mathbf{z}}$. This scenario is graphically depicted in the following figures, where the shaded regions reflect the areas in which the point $\mathbf{z}$ will lie.
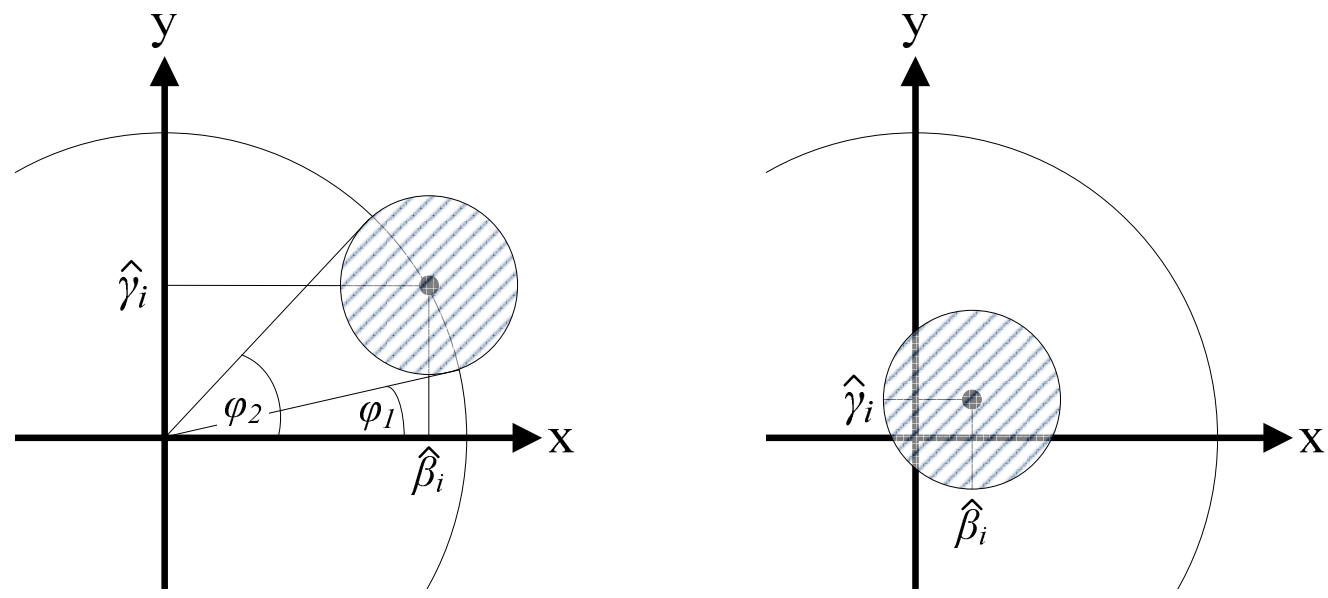
Then, for the first case, the phase of $\mathbf{z}$ will lie between $\phi_{1}$ and $\phi_{2}$ with probability 1 . However, in the second case, where the amplitude is decreased, the phase of $\mathbf{z}$ will lie between $\phi_{1}$ and $\phi_{2}$ with some probability less than 1 , since in general, the estimated phase could take on any value between 0 and $2 \pi$. Furthermore, as an extreme case, when the amplitude of $\hat{\mathbf{z}}$ is 0 (see the figure below), there could be complete uncertainty in the phase of $\mathbf{z}$. This scenario is captured by Eq. (14), in which, as $a_{i}$ approaches 0 , the variance of $\hat{\phi}_{i}$ goes to infinity.

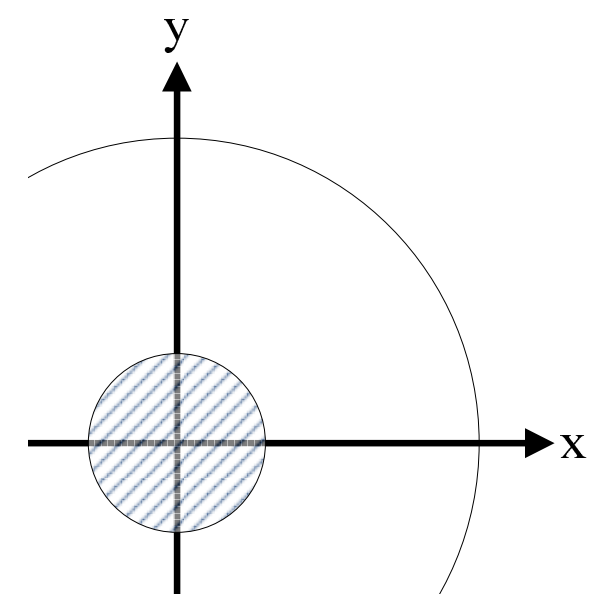

In practice, typically continuous probability density functions are encountered for the estimates $\hat{\beta}_{i}$ and $\hat{\gamma}_{i}$, where the probability density function is nonzero over the entire XY plane. However, the finite distribution model of Example 3 is helpful for gaining an understanding of the statistical phenomenon when converting uncertainty from Cartesian variables to polar variables. 